\title{
Fast summation of divergent series and resurgent transseries from Meijer- $G$ approximants
}

\author{
Héctor Mera, ${ }^{1,2,3}$, Thomas G. Pedersen, ${ }^{2,3}$ and Branislav K. Nikolić ${ }^{1}$ \\ ${ }^{1}$ Department of Physics and Astronomy, University of Delaware, Newark, Delaware 19716-2570, USA \\ ${ }^{2}$ Department of Physics and Nanotechnology, Aalborg University, Aalborg Øst DK-9220, Denmark \\ ${ }^{3}$ Center for Nanostructured Graphene $(C N G)$, Aalborg $\emptyset_{\text {st }}$ DK-9220, Denmark
}

(Received 16 February 2018; published 30 May 2018)

\begin{abstract}
We develop a resummation approach based on Meijer- $G$ functions and apply it to approximate the Borel sum of divergent series and the Borel-Écalle sum of resurgent transseries in quantum mechanics and quantum field theory (QFT). The proposed method is shown to vastly outperform the conventional Borel-Padé and Borel-Padé-Écalle summation methods. The resulting Meijer- $G$ approximants are easily parametrized by means of a hypergeometric ansatz and can be thought of as a generalization to arbitrary order of the Borel-hypergeometric method [Mera et al., Phys. Rev. Lett. 115, 143001 (2015)]. Here we demonstrate the accuracy of this technique in various examples from quantum mechanics and QFT, traditionally employed as benchmark models for resummation, such as zero-dimensional $\phi^{4}$ theory; the quartic anharmonic oscillator; the calculation of critical exponents for the $N$-vector model; $\phi^{4}$ with degenerate minima; self-interacting QFT in zero dimensions; and the summation of one- and two-instanton contributions in the quantum-mechanical double-well problem.
\end{abstract}

DOI: 10.1103/PhysRevD.97.105027

\section{INTRODUCTION}

Perturbative expansions in quantum mechanics, quantum field theory (QFT), and string theory often have zero radius of convergence; i.e., they are asymptotic [1,2]. Optimal truncation of such series to a small number of terms can provide experimentally relevant results but only at a sufficiently small coupling constant. This is exemplified by the asymptotic series for the LoSurdo-Stark effect of atoms and molecules where first- and second-order terms match measurements well, but only for very weak electric fields [3], or by high precision calculations of multiloop Feynman diagrams in quantum electrodynamics [4]where the fine-structure constant is small. On the other hand, extracting physically relevant information from the asymptotic series at larger coupling constants calls, almost invariably, for resummation techniques as exemplified by the LoSurdo-Stark effect $[5,6]$ and field assisted excitonic ionization in layered materials [7], anharmonic oscillators in quantum mechanics [8,9], $\phi^{4}$ theory in QFT [10], quantum chromodynamics [11], string perturbation theory $[12,13]$, and diagrammatic Monte Carlo techniques in

\footnotetext{
*hypergeometric2f1@gmail.com
}

Published by the American Physical Society under the terms of the Creative Commons Attribution 4.0 International license. Further distribution of this work must maintain attribution to the author(s) and the published article's title, journal citation, and DOI. Funded by SCOAP. condensed matter physics [14]. Given the ubiquity of divergent series in physics, research on summation techniques remains an active research area $[15,16]$.

Conventional resummation is, however, not sufficient in the presence of the so-called Stokes phenomenon where different asymptotic expansions hold in different regions of the plane made up of complexified expansion parameter values [17-23]. Thus the Stokes phenomenon requires generally distinct resummations in each of these regions. This complexity is captured by resurgent transseries [21,24-28], which include both analytic polynomial terms and nonanalytic exponential and logarithmic terms. In principle, resurgent transseries offer a nonperturbative framework to reconstruct the original function, which has led to recent vigorous efforts to examine their promise in physically relevant examples, where different sectors are generated by nonperturbative semiclassical effects such as instantons $[22,23,28]$.

Nonetheless, the resurgent transseries also need to be resummed in order to obtain a sensible result. However, the conventional Borel-Padé-Écalle resummation used for this purpose typically requires a large number of terms $[23,29]$ from each sector in order to obtain reasonably accurate results beyond the weak coupling regime. This makes it useless for problems in QFT $[4,10]$ or many-body perturbation theory in condensed matter physics [14] where $\lesssim 10$ orders are available at best. In such cases the Borel-Padé (BP) technique can be used together with a conformal transformation in the Borel plane [1,10,30-37]. 
An alternative is to use an optimized or variational perturbation theory [38] where approximants are equipped by one or more variational parameters, and the approximants that depend the least on these parameters are chosen as optimal (principle of least sensitivity in approximation theory) [39]. In this sense it is worth pointing out the work of Kompaniets and Panzer [10] that epitomizes state-of-theart resummation in the face of the incomplete information represented by the several coefficients that can actually be computed: these coefficients generate a vast approximant space; additional information (such as minimal sensitivity, known bounds on the function and its derivatives) needs to be used in order to narrow down the approximant space toward the physical result. We emphasize that conformal mapping technique and variational perturbation theory can reach high accuracy, but lack the algorithmic simplicity that make techniques such as Padé and Borel-Padé so popular and widely used.

Here we introduce a new algorithm that replaces the standard Padé approximants in the Borel plane by more general and flexible hypergeometric functions (of, in principle, arbitrarily high order), thereby achieving great convergence acceleration toward the exact sum of divergent series and resurgent transseries. Thus, our approach effectively replaces the conventional Borel-Padé and BorelPadé-Écalle summation by Borel-hypergeometric and Borel-hypergeometric-Écalle summation whose approximants admit a representation in terms of Meijer- $G$ functions that are easily parametrized.

Prior to going into technical details, we highlight the power of our algorithm by noting that our approximants converge to the exact nonperturbative, ambiguity-free, partition function for zero-dimensional self-interacting QFT with just five orders, whereas Borel-Padé-Écalle resummation in Ref. [29] needed tens of terms to find a good approximation at intermediate coupling strengths. In practice only a handful of expansion coefficients are typically available: for instance the $\epsilon$ expansion for the $O(n)$-symmetric $\phi^{4}$ theory is only known to six-loop order [10], while the five-loop QCD beta function and anomalous dimension have been calculated only very recently [40]. It is then clear that, for a summation technique to be practical, it needs to be able to return accurate estimates of the sum of a divergent series with only a few coefficients. The highaccuracy at low orders of the Meijer- $G$ approximants introduced in this work makes them suitable for practical applications.

The paper is organized as follows. In Sec. II we introduce the algorithm to transform the expansion coefficients of a divergent expansion into a table of Meijer- $G$ approximants. In Sec. III we apply our algorithm to sum three well-known examples of Borel-summable divergent series in QFT and quantum mechanics: zero-dimensional $\phi^{4}$ theory, the quartic anharmonic oscillator, and the three-dimensional self-avoiding walk case in the $N$ vector model. In Sec. IV we turn our attention to Borel-Écalle summation of resurgent transseries, considering $\phi^{4}$ theory with degenerate minima, self-interacting zero-dimensional QFT, and finally the resummation of the one- and two-instanton contributions in the double-well problem of quantum mechanics. While in Sec. III we deal with Borel-summable divergent expansions, the cases considered in Sec. IV offer an opportunity to demonstrate the efficiency of our approach for the summation of transseries/multi-instanton expansions, i.e., those cases where the perturbation expansion is not Borel-summable due to obstructions (such as poles or branch cuts) along the positive real axis [13]. The Meijer- $G$ summation method is shown to work well in all of these cases, providing a fast way to evaluate the Borel sum of a divergent series and massively outperforming the Borel-Padé and Borel-Padé-Écalle approaches. In some of these examples the Meijer- $G$ approximants converge to the exact result at five-loop order, while in the other cases the convergence is slower-although fast when compared to Borel-Padé and Borel-Padé-Écalle approaches. Finally in Sec. V we discuss the advantages and disadvantages of our approach as an alternative to traditional Borel-Padé techniques. We conclude in Sec. VI.

\section{MEIJER-G APPROXIMANTS}

An efficient resummation technique should be capable of taking a handful of coefficients and returning an accurate estimate of the sum of a divergent perturbation series. In this section such a technique is introduced, providing an algorithm that efficiently transforms the coefficients of a divergent perturbation expansion into a table of Meijer- $G$ functions that serve as approximants. For completeness we first briefly review the traditional Borel-Padé resummation, emphasizing the inherent difficulties faced by such a method when it comes to summation "on the cut." We will then briefly review alternatives to Padé and Borel-Padé that make use of analytic continuation functions with a built-in branch cut, in particular the hypergeometric resummation method we introduced in Refs. [5-7]. After reviewing these other approaches we finally introduce the algorithm to calculate Meijer- $G$ approximants and highlight various properties that make this method well suited to yield inexpensive - and yet accurate-low order approximations to the sum of a divergent series.

\section{A. Borel-Padé resummation}

When it comes to sum divergent series, Borel-Padé has become the dominant approach $[1,15,41]$. There are various reasons for the popularity of this approach but it can be argued that the most important of these is its algorithmic simplicity. The Borel-Padé approach is in essence a simple recipe to transform the coefficients of a divergent expansion into a table of approximants, which approximate the Borel sum of a divergent series and are typically evaluated by numerical contour integration. Another advantage of this 
approach is that it relies on widely studied approaches: conditions for Borel summability are rather well understood [41-44], and the properties of Padé approximantsused at a crucial step in the algorithm - are also very well known as they have been studied in depth for decades [45]. Given a divergent expansion $Z(g) \sim \sum_{n=0}^{\infty} z_{n} g^{n}$, where $z_{n}$ are the expansion coefficients and $g$ is the expansion parameter ("the coupling"), the algorithm to calculate the Borel sum of a divergent series can be summarized as follows:

Step 1: Borel transform. Calculate the Borel-transformed coefficients: $b_{n}=z_{n} / n !$.

Step 2: Summation in the Borel plane. Sum the series $B(\tau) \sim \sum_{n=0}^{\infty} b_{n} \tau^{n}$; this series is called the Borel-transformed series. The complex- $\tau$ plane is known as the Borel plane.

Step 3: Laplace transform. The Borel sum of the series, $Z_{B}(g)$, is given by the Laplace transform

$$
Z_{B}(g)=\int_{0}^{\infty} e^{-\tau} B(\tau g) d \tau .
$$

The rationale behind the Borel summation method is simple: the coefficients of a series with zero radius of convergence typically grow factorially at large orders. In the first step such factorial growth is removed and the Borel-transformed series is more tractable since it has a finite nonzero radius of convergence. By summing the Borel-transformed series one finds the function $B(\tau)$, and then the Laplace transform can be calculated numerically to find the Borel sum $Z_{B}(g)$. The Borel-Padé summation method is a practical algorithm to find, in principle, increasingly accurate approximations to the Borel sum. In this approach the second step above is specialized to Padé summation: Step 2: Padé summation in the Borel plane. Use Padé approximants to approximately sum the series $B(\tau) \sim \sum_{n=0}^{\infty} b_{n} \tau^{n}$, from the knowledge of the partial sums to order $N$, i.e., $\sum_{n=0}^{N} b_{n} \tau^{n}$. In Padé summation one approximates $B(\tau)$ by a rational function of $\tau$

$$
B_{L / M}(\tau)=\frac{\sum_{n=0}^{L} p_{n} \tau^{n}}{1+\sum_{n=1}^{M} q_{n} \tau^{n}},
$$

where $L+M=N$. Here the coefficients $p_{n}$ and $q_{n}$ are found by equating order by order the Taylor series of $B_{L / M}(\tau)$ around $\tau=0$ with the asymptotic expansion of $B(\tau)$, up to the desired order. These approximants are then used in step 3 of the algorithm to evaluate the Laplace transform and therefore to find the $L / M$-Borel-Padé approximant to the Borel sum of $Z(g), Z_{B, L / M}(g)$, as

$$
Z_{B, L / M}(g)=\int_{0}^{\infty} e^{-\tau} B_{L / M}(\tau g) d \tau .
$$

Clearly the Borel-Padé method returns a table of approximants: for instance, by knowing the partial sums to second

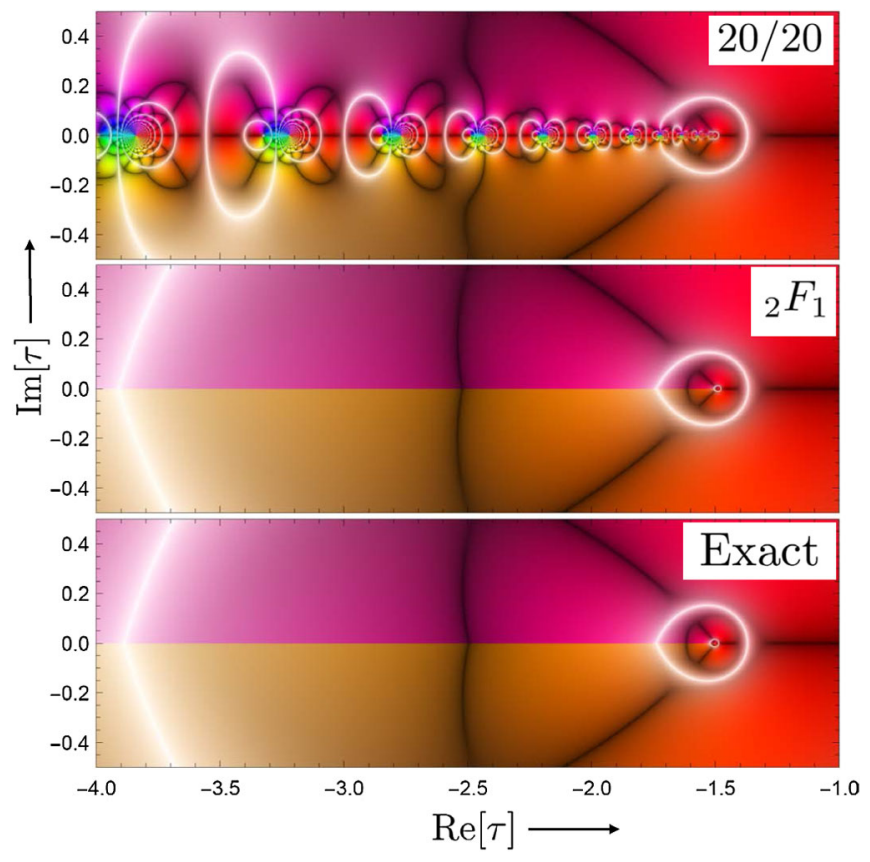

FIG. 1. Domain coloring plot of approximants in the Borel plane calculated for the partition function of $\phi^{4}$ theory in zero dimensions for $g=1$. Branch cuts are represented by a discontinuous change of color. Around poles one sees gradual color changes, intersecting black lines and concentric white lines. Top panel: 20/20 Padé approximant. Middle panel: hypergeometric ${ }_{2} F_{1}$ approximant. Bottom panel: Exact. The Padé approximant mimics the exact branch cut by placing poles next to each other and high orders are needed to accurately model the cut. In contrast, the third-order ${ }_{2} F_{1}$ approximant with a built-in branch cut very nearly reproduces the exact branch cut.

order $(N=2)$ one can calculate $B_{1 / 0}, B_{0 / 1}, B_{2 / 0}, B_{1 / 1}$, and $B_{0 / 2}$.

There are, however, various types of problems for which Borel-Padé approximants are not well suited. Padé approximants are rational functions whose built-in singularities are poles. It is well known that in many situations the convergence of the Borel-transformed series is limited by the branch point singularity closest to the origin, the terminal point of a dense line of poles known as a branch cut. In such scenarios Borel-Padé approximants can converge very slowly since many poles may be needed to properly mimic a branch cut. Thus, to overcome this difficulty with Borel-Padé one has to replace Padé approximants, adopting instead as approximants functions that are able to mimic branch cuts in the Borel plane. The advantages of that strategy are highlighted in Fig. 1 where we show a domain coloring plot of a function $B(\tau)$ in the Borel plane (corresponding to the partition function of zero-dimensional $\phi^{4}$ for $g=1$ ). We show the 20/20 Padé approximant, $B_{20 / 20}(\tau)$ (top panel), together with the exact $B(\tau)$ (bottom panel). We also show a hypergeometric ${ }_{2} F_{1}$ approximant (middle panel) in the Borel plane which corresponds to the third-order Meijer- $G$ approximant, 
introduced in Sec. II C. The Padé approximant in the Borel plane attempts to reproduce the branch cut by brute forceplacing poles next to each other along the negative $\tau$ axis. The Meijer- $G$ approximant has a built-in branch cut in the Borel plane, and it can thus very accurately mimic the branch cut using only three orders of perturbation theory. One has to carefully look at the details in Fig. 1 to see the very minor differences between the exact $B(\tau)$ and its thirdorder hypergeometric approximant.

\section{B. Hypergeometric resummation}

Recently we have introduced hypergeometric resummation-a technique that enables summation on the cut using only a small number of expansion coefficients [5-7,46,47]. Various flavors of this technique were applied to a variety of problems with good results: in particular, it was shown how one could use low order data to derive accurate approximations to the decay rate in Stark-type problems [5-7]. Typically the idea is to use hypergeometric functions to analytically continue divergent series. Originally the technique was devised by noticing the shortcomings of Padé approximants when applied to problems in nanoelectronics modeling within the nonequilibrium Green's function theory [48-51]. As pointed out in Sec. II A one seeks to substitute Padé approximants by more general functions that are equipped with branch cuts and can work well in those cases where Padé approximants do not work or converge very slowly. Hypergeometric functions are particularly promising as they are endowed with a branch cut and generalize geometric series (which can be summed exactly by Padé approximants). For instance, given a divergent function $Z(g) \sim \sum_{n=0}^{\infty} z_{n} g^{n}$, with normalized zeroth-order coefficient $z_{0}=1$, one can attempt to find a hypergeometric function $[52,53],{ }_{2} F_{1}\left(h_{1}, h_{2}, h_{3}, h_{4} g\right)$, such that

$$
Z(g)={ }_{2} F_{1}\left(h_{1}, h_{2}, h_{3}, h_{4} g\right)+O\left(g^{5}\right) .
$$

Similar ideas were already considered in Refs. [41,54-56], where the authors used it as part of an algorithm to find convergent strong coupling expansions (from divergent weak coupling ones). Similarly, in Refs. [57,58], the authors used products of hypergeometric functions as approximants. Foreshadowing all of these works are contributions by Stillinger and co-workers [59,60], where early Borel-hypergeometric approximants (typically ${ }_{1} F_{0}$ hypergeometric functions in the Borel plane) are considered, as well as early versions of self-similar factor approximants and exponentialBorel approximants. Of the works mentioned, the work of Stillinger is the closest in philosophy to our own work, as he also considers the ratio test of series convergence as a starting point—as we do in Sec. III C and we did in earlier work [46].

The hypergeometric approximants we introduced in Refs. [5-7,46] have a number of clear limitations, which we overcome in this work and which we enumerate below:
(1) Hypergeometric resummation is uncontrolled: Hypergeometric ${ }_{2} F_{1}$ approximants of third, fourth, and fifth order can be constructed in various ways, but in Refs. [5-7,46] we did not give a recipe for constructing a table of hypergeometric approximants. How does one parametrize and build, e.g., a 21st-order hypergeometric approximant? In order to have control over any approximations one develops, it is fundamental to be able to increase the order of the approximation and to study the convergence properties of the approximants. In this work we provide a set of approximants that can, in principle, be computed at any order.

(2) Difficult parametrization at large orders: A natural attempt to provide a generalization to arbitrary orders would be to state that general hypergeometric functions ${ }_{q} F_{p}[52,53]$ constitute the approximant space. These functions contain $p+q+1$ parameters, ${ }_{p} F_{q}\left(h_{1}, \ldots, h_{p} ; h_{p+1}, \ldots, h_{p+q}, h_{p+q+1} \tau\right)$, that need to be calculated by equating each order of the asymptotic expansion that one seeks to sum with the corresponding order of the expansion of the hypergeometric approximant. However, one faces a degeneracy problem as all the hypergeometric functions obtained by permuting elements of each of the parameter sets $\left(h_{1}, \ldots, h_{p}\right)$ and $\left(h_{p+1}, \ldots, h_{p+q}\right)$ are one and the same. Hence when determining the parameters $h_{i}$ the computational time grows factorially with order - there is a factorially large number of solutions, all of which correspond to the same hypergeometric function. So calculations at the sixloop order are already very expensive and nearly impossible at the seventh-loop order. The same problem is found with other approximants; for instance, self-similar factor approximants [57,58] have rarely been computed beyond the sixth-loop order. In this work we put forward an algorithm that enables fast parametrization of hypergeometric approximants in the Borel plane, at arbitrarily high orders.

(3) Inaccuracies for expansions with zero radius of convergence: It was noted that for very small couplings the hypergeometric approximants (as described in Ref. [5]) gave inaccurate results. While these inaccuracies were exponentially small, they were conceptually important. The reason for them was that the radius of convergence of hypergeometric ${ }_{p+1} F_{p}$ functions is not zero, while we were applying the hypergeometric approximants to problems with zero radius of convergence. For instance, an approximant given by Eq. (4) above has a radius of convergence $g_{c}=1 / h_{4}$. When applied to series with zero radius of convergence the value of $h_{4}$ was typically found to be very large but finite. Therefore the hypergeometric approximants had a Taylor series with a tiny, but nonzero, radius of convergence. 
Hence, in Refs. [6,7] we used a different parametrization of the approximants, which positioned the tip of the hypergeometric branch cut exactly at the origin and therefore alleviated this problem. However, there we did not come up with a clear approach to compute similar approximants of higher order. The approximants derived in this work can have zero radius of convergence, therefore alleviating this difficulty found in our previous approach.

Clearly there is a wide variety of problems for which Borel-Padé resummation can be improved. However, attempts to improve it can easily fall into several pitfalls. In the case of hypergeometric resummation these were difficulties in both extending the approach to arbitrarily high orders and dealing with series with zero radius of convergence. These difficulties are largely surpassed by the Meijer- $G$ approximants we introduce next.

\section{Meijer- $G$ resummation}

We now present an algorithm to transform the low order coefficients of a divergent perturbation expansion, $Z(g) \sim$ $\sum_{n=0}^{\infty} z_{n} g^{n}$ (with normalized coefficients, $z_{0}=1$ ), into a table of approximants to its Borel sum. The algorithm consists of four easy steps. For ease of presentation, in this work we will compute mostly odd-order approximants, giving a short description of the algorithm to compute evenorder approximants below.

Step 1: Borel transform. Imagine that we know only $N$ coefficients, $z_{0}, z_{1}, \ldots, z_{N}$. In this step we compute the Borel-transformed coefficients $b_{n}=z_{n} / n$ ! together with $N$ ratios of consecutive Borel-transformed coefficients, $r(n)=b_{n+1} / b_{n}$. Here we will assume that $N$ is an odd number.

Step 2: Hypergeometric ansatz. We make the ansatz that $r(n)$ is a rational function of $n$. Thus we define a rational function of $n, r_{N}(n)$, as

$$
r_{N}(n)=\frac{\sum_{m=0}^{l} p_{m} n^{m}}{1+\sum_{m=1}^{l} q_{m} n^{m}},
$$

where $l=(N-1) / 2$ and the $N$ unknown parameters $p_{m}$ and $q_{m}$ are uniquely determined (in some case, up to an arbitrary constant) by the $N$ input ratios by means of $N$ equations,

$$
r(n)=\frac{b_{n+1}}{b_{n}}=r_{N}(n), \quad 0 \leq n \leq N-1,
$$

provided that a solution exists. It should be noted that this is a system of $N$ linear equations with $N$ unknowns $\left(q_{m}\right.$ and $\left.p_{m}\right)$ which can easily be solved by a computer. The hypergeometric ansatz is the crucial step that allows an extremely fast parametrization of large order Meijer- $G$ approximants.

Step 3: Hypergeometric approximants in the Borel plane. In this step we undertake the parametrization of hypergeometric approximants in the Borel plane. To do this for $N>1$, we use the calculated $p_{m}$ and $q_{m}$ to set two equations

$$
\begin{aligned}
\sum_{m=0}^{l} p_{m}(x)^{m} & =0, \\
1+\sum_{m=1}^{l} q_{m}(y)^{m} & =0,
\end{aligned}
$$

which yield two solution vectors $\left(x_{1}, \ldots, x_{l}\right)$ and $\left(y_{1}, \ldots, y_{l}\right)$. We refer to these vectors as hypergeometric vectors. It follows from the definition of hypergeometric functions that the hypergeometric vectors determined in this way uniquely determine the hypergeometric function

$$
B_{N}(\tau) \equiv{ }_{l+1} F_{l}\left(\mathbf{x}, \mathbf{y}, \frac{p_{l}}{q_{l}} \tau\right),
$$

where $\mathbf{x}=\left(1,-x_{1}, \ldots,-x_{l}\right), \mathbf{y}=\left(-y_{1}, \ldots,-y_{l}\right)$, and ${ }_{l+1} F_{l}$ is a generalized hypergeometric function [52]. The function $B_{N}(\tau)$ is the hypergeometric approximant in the Borel plane; it provides an $N$ th-order approximation to the sum of the Borel-transformed series. Thanks to the hypergeometric ansatz and the hypergeometric vector equations, the function $B_{N}(\tau)$ can easily be parametrized for arbitrary large $N$.

Step 4: Meijer-G approximants. In this last step we need to reinstate the $n$ ! removed from the expansion coefficients by means of the Borel transform. This is achieved by means of the Laplace transform

$$
Z_{B, N}(g) \equiv \int_{0}^{\infty} e^{-\tau} B_{N}(g \tau) d \tau,
$$

which gives the desired approximation to the Borel sum of the asymptotic expansion of $Z(g)$ in the complexified $g$ plane. This expression admits the representation

$Z_{B, N}(g)=\frac{\prod_{i=1}^{l} \Gamma\left(-y_{i}\right)}{\prod_{i=1}^{l} \Gamma\left(-x_{i}\right)} G_{l+1, l+2}^{l+2,1}\left(\begin{array}{c}1,-y_{1}, \ldots,-y_{l} \\ 1,1,-x_{1}, \ldots,-x_{l}\end{array} \mid-\frac{q_{l}}{p_{l} g}\right)$,

where $\Gamma(x)$ is Euler's Gamma function and $G_{p, q}^{m, n}\left(\begin{array}{l}a_{1}, \ldots, a_{p} \\ b_{1}, \ldots, b_{q}\end{array} \mid z\right)$ is Meijer's $G$ function [52,61,62]. This algorithm then transforms $N$ available input coefficients $z_{n}$ into a table of Meijer- $G$ functions, which approximate the Borel sum of $Z(g)$. We once again emphasize how easily one can parametrize these extremely complex functions: all that was needed was the hypergeometric ansatz and the resulting hypergeometric vectors. Once these are determined the Meijer- $G$ approximants can be parametrized for arbitrarily large orders.

There are various remarks that we would like to add before moving onto the practical application of this method. The even-order approximants can be computed in exactly the same way, but one starts from a once-subtracted series; for instance, from the once-subtracted Borel-transformed series 
$\left(\sum_{n=0}^{\infty} b_{n} \tau^{n}-1\right) /\left(b_{1} \tau\right)$ and the $B_{N}$ are calculated as above but with $l=N / 2$. The Laplace transform then gives the even-order approximants. It is also easy to compute approximations to the Mittag-Leffler (ML) sum [63], which is a generalization of the Borel sum where one can remove superfactorial asymptotic coefficient growth (as opposed to factorial) by using a ML transform of the coefficients (as opposed to a Borel transform); in step 1 the ML-transformed coefficients are $b_{n}=a_{n} / \Gamma(\alpha n+\beta)$, where $\alpha$ and $\beta$ are real numbers, and the odd approximants in step 4 are given by $Z_{B, N}(g)=\int_{0}^{\infty} e^{-\tau} \tau^{\beta-1+\alpha} B_{N}\left(g \tau^{\alpha}\right) d \tau$. When $\alpha=\beta=1$, ML summation is equivalent to Borel summation. These approximants also admit a Meijer- $G$ function representation (not shown). Furthermore, generalizations of Borel and ML summation methods where essentially arbitrary asymptotic coefficient growth is removed can easily be arrived at.

On the practical side it is important to note that in some cases there might be no solutions to the hypergeometric vector equations in step 3 of our algorithm. An example is given at the end of Sec. III A. As we will see below, there are cases (shown is Secs. III A, IV A, and IV B) where the Borel transformed series is actually hypergeometric and the approximants converge. In those cases the coefficients $p_{m}$ and $q_{m}$ are determined up to an arbitrary constant.

We would like to point out the domain of applicability of our approximants. The idea behind the hypergeometric ansatz is the well-known ratio test of series convergence: if the ratios between consecutive coefficients $r(n)$ goes to a constant $C$ as $n \rightarrow \infty$, then the radius of convergence is $1 / C$. One can approximate these ratios by a rational function of the form given by $r_{N}(n)$ in Eq. (5), satisfying $\lim _{n \rightarrow \infty} r_{N}(n)=p_{l} / q_{l}=1 / C$. Our choice of $r_{N}(n)$ is then a symmetric rational function, with same-order polynomials in numerator and denominator. If the polynomial in the numerator were of higher order than the one in the denominator, then the rational function would mimic the ratios of a divergent series since $C=0$, and the resulting summation would yield a divergent hypergeometric series, which is still divergent. Hence, such a choice of rational function does not solve the problem. Here we assume that the Borel transform (or more general transforms to be discussed elsewhere) removes all asymptotic coefficient growth, and therefore $C$ is finite; this explains our choice of rational function. On the other hand, one could choose instead a rational function where the polynomial in the denominator is of higher order than that of the numerator; but in such a case the Borel-transformed series would have infinite radius of convergence and the approximants in the Borel plane would be diagonal hypergeometric functions, which nevertheless can be thought of as limiting cases of our ${ }_{l+1} F_{l}$ [52].

We emphasize that steps 2 and 3 of our algorithm are themselves a recipe to find hypergeometric approximants to series with finite (nonzero) radius of convergence. Because of the very general nature of the hypergeometric functions listed in Eq. (8), which form a massive approximant space encompassing very many special functions as particular cases, we expect such approximants to be highly accurate and rapidly convergent in those cases where the convergence is limited by a single branch cut (hypergeometric functions contain only one branch cut [52]) or in those cases with two equally distant cuts, where the expansion contains only odd or only even powers of the coupling.

The observations from the previous paragraph can be translated to the Borel plane. Our method should work well for cases where the convergence of the Borel-transformed series is limited by a single branch cut. As we have emphasized in Secs. II A and II B, approximants able to efficiently deal with these kinds of series are very much needed. Finally, it follows from the differential equation satisfied by Meijer- $G$ functions that the Meijer- $G$ approximants given by Eq. (10) provide a regularizing analytic continuation of the divergent hypergeometric functions ${ }_{l+2} F_{l}\left(-y_{1}, \ldots,-y_{l} ; 1,1,-x_{1}, \ldots,-x_{l} ; \frac{p_{l} \lambda}{q_{l}}\right)[61]$. Such hypergeometric functions have zero radius of convergence; the fact that Meijer- $G$ approximants are able to "sum" them clearly illustrates the potential of these functions for the summation of divergent series.

\section{SUMMATION OF DIVERGENT SERIES}

In this section Meijer- $G$ approximants are used to sum various examples of Borel-summable divergent perturbation theory in physics. In particular, in Sec. III A we will consider the summation of the partition function in $\phi^{4}$ theory, while in Secs. III B and IIIC we consider the calculation of the ground-state energy of the quantummechanical quartic anharmonic oscillator and the resummation of the critical exponents for self-avoiding walks in three dimensions, respectively. We shall see that for the first of these examples our summation procedure converges and that we are thus able to find a Meijer- $G$ function representation for the partition function directly from the coefficients of its perturbation expansion. This is so because the Borel-transformed series is a hypergeometric series for which the hypergeometric ansatz is exact. For the quartic anharmonic oscillator and the critical exponents the Borel-transformed series is not exactly hypergeometric; nevertheless our approximants return excellent approximations for these quantities, which can be systematically improved by adding more terms to the expansion.

\section{A. Partition function in $\phi^{4}$ theory}

The partition function in zero-dimensional $\phi^{4}$ theory is given by

$$
Z(g)=\sqrt{\frac{2}{\pi}} \int_{0}^{\infty} e^{-\phi^{2} / 2-g \phi^{4} / 4 !} d \phi,
$$

which for $\operatorname{Re}[g]>0$ can be written as 


$$
Z(g)=\sqrt{\frac{3}{2 \pi g}} e^{\frac{3}{4 g}} K_{\frac{1}{4}}\left(\frac{3}{4 g}\right),
$$

where $K_{\nu}(x)$ is a modified Bessel function of the second kind. This partition function is commonly used to benchmark resummation techniques-see Refs. [15,64] for two recent examples.

The first few terms of the asymptotic expansion about $g=0 \mathrm{read}$

$$
Z(g) \sim 1-\frac{1}{8} g+\frac{35}{384} g^{2}-\frac{385}{3072} g^{3}+\frac{25025}{98304} g^{4}+\cdots .
$$

The expansion coefficients grow factorially at large orders, and thus this expansion has zero radius of convergence. Here the calculation of $Z(g)$ by direct resummation of the asymptotic expansion serves two main purposes. On the one hand, it provides a simple test system for benchmarking against Borel-Padé. On the other hand, the parametrization of the approximants can be performed analytically at low orders by following our algorithm and thus is valuable from a didactical perspective.

Let us calculate the first-order and third-order Meijer- $G$ approximant for this problem analytically, by running the above-given algorithm explicitly. We start with the firstorder calculation and proceed step by step.

(1) Borel transform: We calculate the Borel transformed coefficients $b_{n}=z_{n} / n$ !. In a first-order calculation we have just two coefficients, $b_{0}=1$ and $b_{1}=-1 / 8$.

(2) Hypergeometric ansatz: We compute the ratios between consecutive coefficients as a function of $n$, $r(n)$, and we approximate the ratio by a rational function of $n$. In this case we have only one ratio $r(0)=b_{1} / b_{0}=-1 / 8$, and the rational function that approximates $r(n)$ is just a constant: $r(n) \approx r(0), \forall n$.

(3) Hypergeometric approximant in the Borel plane: In this case the hypergeometric vectors are empty, $\left(x_{1}, \ldots, x_{l}\right)=\{\}$ and $\left(y_{1}, \ldots, y_{l}\right)=\{\}$ and therefore $\mathbf{x}=1$ and $\mathbf{y}=\{\}$. Since $l=(N-1) / 2=0$ for $N=1$, the first-order hypergeometric approximant in the Borel plane is given by

$$
B_{H, N=1}(\tau)={ }_{1} F_{0}(1, r(0) \tau) .
$$

This ${ }_{1} F_{0}$ hypergeometric function is just the $0 / 1$ Padé approximant to the Borel-transformed series, i.e.,

$$
B_{H, 1}(\tau)=\frac{1}{1+r(0) \tau}
$$

It should be clear that the hypergeometric vectors are empty, and that the above-given hypergeometric function in the Borel plane reduces to the geometric case. Therefore our first-order hypergeometric approximant coincides with the $0 / 1$ Padé approximant.

(4) Meijer-G approximant: Once the hypergeometric approximant in the Borel plane is found, we can use Eq. (10) to immediately write down the corresponding Meijer- $G$ approximant in the complexified- $g$ plane, which reads

$$
Z_{B, 1}(g)=\frac{8}{g} G_{1,2}^{2,1}\left({ }_{0,0}^{0} \mid \frac{8}{g}\right) .
$$

This Meijer- $G$ approximant is just the $0 / 1$ BorelPadé approximant. This is an interesting aspect of hypergeometric and Borel-hypergeometric resummation: to first-order hypergeometric approximants are just 0/1 Padé approximants; translating this observation to the Borel plane shows that the corresponding Meijer- $G$ (Borel-hypergeometric) approximant is just the $0 / 1$ Borel-Padé approximant. Indeed,

$$
\begin{aligned}
Z_{B, 1}(g) & =\int_{0}^{\infty} \frac{e^{-\tau}}{1+r(0) \tau} d \tau \\
& =-\frac{1}{r(0) g} U\left(1,1,-\frac{1}{r(0) g}\right),
\end{aligned}
$$

where $U(a, b, x)$ is Tricomi's hypergeometric $U$ function [52], is both the 0/1 Borel-Padé approximant and the first-order Meijer- $G$ approximant.

The conclusion from this first example calculation is that our first-order Borel-hypergeometric (Meijer-G) approximant is the first-order Borel-Padé approximant. This illustrates a general property of Meijer- $G$ approximations to the Borel sum: their first order is just identical to a firstorder Borel-Padé approximant. The interested reader can now look to the closely related approach put forward in Ref. [15] — both approaches take very different routes beyond first order.

Next we run again our algorithm to obtain the third-order approximant. While the first-order Meijer- $G$ approximant is identical to the first-order Borel-Padé approximant we will see shortly that the third-order Meijer- $G$ approximant is substantially more accurate than Borel-Padé approximants of the same and much higher orders.

(1) Borel transform. In this case we have four Boreltransformed coefficients: $b_{0}=1, \quad b_{1}=-1 / 8$, $b_{2}=35 / 768$, and $b_{3}=-385 / 18432$.

(2) Hypergeometric Ansatz. Here we have three ratios: $r(0)=-1 / 8, r(1)=-35 / 96$, and $r(3)=-11 / 24$. We approximate $r(n)$ as $r(n)=r_{3}(n)$ where

$$
r_{3}(n)=\frac{p_{0}+p_{1} n}{1+q_{1} n},
$$

and use the known ratios, $r(0), r(1)$, and $r(2)$ to find $p_{0}, p_{1}$, and $q_{1}$ by requiring 


$$
r(n)=r_{3}(n), \quad n=0,1,2
$$

which leads to three equations

$$
\begin{gathered}
r(0)=-\frac{1}{8}=p_{0}, \\
r(1)=-\frac{35}{96}=\frac{p_{0}+p_{1}}{1+q_{1}}, \\
r(2)=-\frac{11}{24}=\frac{p_{0}+2 p_{1}}{1+2 q_{1}},
\end{gathered}
$$

with three unknowns, $p_{0}, p_{1}$, and $q_{1}$. These equations yield a solution

$$
p_{0}=-\frac{1}{8}, \quad p_{1}=-\frac{113}{216}, \quad q_{1}=\frac{7}{9} .
$$

Therefore our third-order rational approximation to the ratios $r(n)$ reads

$$
r(n) \approx r_{3}(n)=\frac{-1 / 8-113 n / 216}{1+7 n / 9}
$$

(3) Hypergeometric approximant in the Borel plane. To build the hypergeometric approximants in the Borel plane we need to find the hypergeometric vectors, that is, to find the values of $x$ and $y$ that solve these two equations:

$$
\begin{gathered}
p_{0}+p_{1} x=0, \\
1+q_{1} y=0,
\end{gathered}
$$

which yield $x_{1}=-p_{0} / p_{1}$ and $y_{1}=-1 / q_{1}$ and thus find the vectors $\mathbf{x}=\left(1, p_{0} / p_{1}\right)$ and $\mathbf{y}=\left(-1 / q_{1}\right)$. Hence the third-order hypergeometric approximant in the Borel plane is

$$
B_{H, 3}(\tau)={ }_{2} F_{1}\left(1, \frac{p_{0}}{p_{1}}, \frac{1}{q_{1}}, \frac{p_{1}}{q_{1}} \tau\right) .
$$

Substituting values of $p_{i}$ and $q_{i}$ we get

$$
B_{H, 3}(\tau)={ }_{2} F_{1}\left(1, \frac{27}{113}, \frac{9}{7},-\frac{113}{168} \tau\right) .
$$

(4) Meijer- $G$ approximant. We read off the Meijer- $G$ approximant directly from the hypergeometric vectors to get a third-order approximant that can be compactly written as

$$
Z_{B, 3}(g)=\frac{\Gamma(9 / 7)}{\Gamma(27 / 113)} G_{2,3}^{3,1}\left(\begin{array}{c|c}
1,9 / 7 & 168 \\
1,1,27 / 113 & 113 g
\end{array}\right.
$$

One can easily calculate higher order Meijer- $G$ approximants. It turns out that the fifth-order Meijer- $G$ approximant is converged and equal to the exact Borel sum, i.e.,

$$
Z_{B, 5}(g)=Z(g)
$$

and all higher order Meijer- $G$ approximants are also equal to $Z(g)$, i.e.,

$$
Z_{B, 5}(g)=Z_{B, 7}(g)=\cdots=Z(g)
$$

What is happening is that the rational approximations used in the hypergeometric ansatz have converged at fifth order; i.e., the ratio between consecutive Borel-transformed coefficients is indeed a rational function of $n$, and, in fact, of the form

$$
r(n)=\frac{p_{0}+p_{1} n+p_{2} n^{2}}{1+q_{1} n+q_{2} n^{2}},
$$

specifically

$$
r(n)=\frac{-1 / 8-2 n / 3-2 n^{2} / 3}{1+2 n+n^{2}}
$$

which reproduces the ratios between Borel-transformed coefficients up to arbitrarily high orders. Approximating these ratios by rational functions of higher order, such as $r_{7}(n)$, one finds the same rational function once again. Hence the approximants of order five or higher are converged.

We now compare the performance of Meijer- $G$ approximants with that of Borel-Padé approximants. In Fig. 2 we compare the 5/5, 10/10, and 20/20 Borel-Padé approximants (of orders 10,20, and 40, respectively) with the third and fifth-order Meijer- $G$ approximant for large values of $g$. It is clear that the third-order Meijer- $G$ approximant is more accurate than the 10/10 Borel-Padé approximant, but less accurate than the 40-order 20/20 Borel-Padé approximant. The fifth-order Meijer- $G$ approximant is exact, and it is therefore more accurate than any Borel-Padé approximant.

It is instructive to compare $Z(g)$ with $Z_{3}(g)$ for $g<0$. For instance,

$$
Z(-10+\mathrm{i} \epsilon)=0.7463895836-\mathrm{i} 0.4368446698,
$$

while

$$
Z_{3}(-10+\mathrm{i} \epsilon)=0.7443450750-\mathrm{i} 0.4362172724,
$$




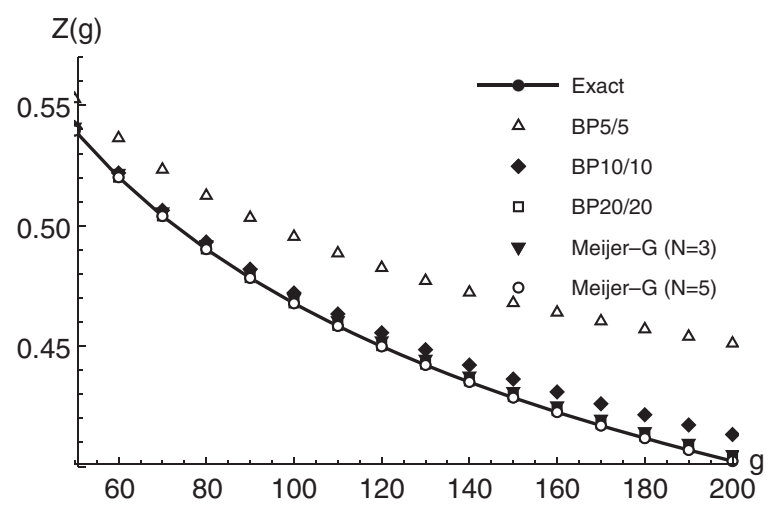

FIG. 2. $Z(g)$ for zero-dimensional $\phi^{4}$ theory calculated using Meijer- $G$ and Borel-Padé approximants. For large values of $g$ the third-order Meijer- $G$ approximant (filled inverted triangles) and the fifth-order Mejer- $G$ approximant (empty circles) are compared with higher order 5/5 (empty triangles), 10/10 (filled diamonds), and 20/20 (empty squares) Borel-Padé approximants and with the exact $Z(g)$ (solid line with filled circles). The thirdorder Meijer- $G$ approximant is more accurate that $5 / 5$ and $10 / 10$ Borel-Padé approximants, and slightly less accurate than the 20/20 Borel-Padé approximant. The fifth-order Meijer-G approximant is exact and thus more accurate than any Borel-Padé approximant.

which demonstrates the great accuracy of Meijer- $G$ approximants for rather large negative couplings; in particular, the imaginary part of $Z(g)$ is reproduced within a percent. In Table I we compare again the third- and fifth-order Meijer- $G$ approximants against the Borel-Padé approximants - this time for negative couplings. We see that for $g=-1$ all approximants are very accurate. The third-order Meijer- $G$ approximant is more accurate than the 2/2 Borel-Padé approximant, but less accurate than the other Borel-Padé approximants shown. For $g=-10$ the Meijer- $G$ approximant is already more accurate than $2 / 2,5 / 5$, and $10 / 10$ Borel-Padé approximants. Finally for $g=-100$ the Meijer- $G$ approximant is more accurate than all the Borel-Padé approximants reported. The fifth-order
Meijer- $G$ approximant reproduces the exact result. It should be mentioned that for this problem conformal mapping gives more precise results than Borel-Padé and that it can be combined with the strong-coupling expansions to reproduce the exact result [30].

While Meijer- $G$ approximants can provide an exact reconstruction of $Z(g)$ we note that it is actually possible (and easy) to use this model to build a pathological example, so that there are no solutions to the hypergeometric vector equations. This is done by considering the following modification to $Z(g)$ :

$$
\tilde{Z}(g)=\frac{1+\int_{0}^{\infty} d \phi e^{-\phi^{2} / 2-g \phi^{4} / 4 !}}{1+\sqrt{\pi / 2}},
$$

which satisfies $\tilde{Z}(g=0)=Z(g=0)=1$. The remaining expansion coefficients, $\tilde{z}_{n}$ with $n \geq 1$, are proportional to the expansion coefficients $z_{n}$ of $Z(g)$. Accordingly the ratios between consecutive coefficients of $\tilde{Z}(g)$ are identical to those of $Z(g)$, except for the first one. This in turn means that there cannot be a solution to the hypergeometric vector equations for the case of $\tilde{Z}(g)$, beyond $N=5$. While rather artificial, this example showcases the possibility of problems for which solutions to hypergeometric vector equations may not exist beyond a certain order and sheds light on the importance of trying various subtraction schemes.

\section{B. Quartic anharmonic oscillator}

The quartic anharmonic oscillator exemplifies the divergence of Rayleigh-Schrödinger perturbation theory characteristic of quantum mechanical models [9] and serves as a benchmark system for which exact results are readily available, and on which new resummation techniques are commonly tried and tested. In this section we compare the results of Borel-hypergeometric resummation with those of variational perturbation theory (VPT) [38] and Borel-Padé approximants. We will show that Meijer- $G$ approximants

TABLE I. $Z(g)$ for zero-dimensional $\phi^{4}$ theory evaluated on the cut, for $g=-1, g=-10$, and $g=-100$ using Borel-Padé approximants of orders 4 (BP2/2), 10 (BP5/5), 20 (BP10/10), and 40 (BP20/20), compared with thirdand fifth-order Meijer- $G$ approximants and with the exact value. All results are given to six significant digits. The accuracy of the third-order Meijer- $G$ approximant is comparable to that of BP approximants of higher order at weak couplings $(g=-1)$ and greater at large couplings $(g=-10$ and $g=-100)$. The fifth-order Meijer- $G$ approximant is exact.

\begin{tabular}{lccc}
\hline \hline Method & $g=-1$ & $g=-10$ & $g=-100$ \\
\hline BP2/2 & $1.132752-0.129446 \mathrm{i}$ & $0.598308-0.424956 \mathrm{i}$ & $0.473216-0.070069 \mathrm{i}$ \\
BP5 $/ 5$ & $1.133180-0.144446 \mathrm{i}$ & $0.784563-0.458166 \mathrm{i}$ & $0.300204-0.251866 \mathrm{i}$ \\
BP10/10 & $1.133022-0.144983 \mathrm{i}$ & $0.740363-0.458776 \mathrm{i}$ & $0.329910-0.450161 \mathrm{i}$ \\
BP20/20 & $1.133028-0.144995 \mathrm{i}$ & $0.746175-0.494474 \mathrm{i}$ & $0.402820-0.519661 \mathrm{i}$ \\
Meijer- $G(N=3)$ & $1.133285-0.144952 \mathrm{i}$ & $0.744345-0.436217 \mathrm{i}$ & $0.386356-0.321210 \mathrm{i}$ \\
Meijer- $G(N=5)$ & $1.133029-0.144984 \mathrm{i}$ & $0.746390-0.436845 \mathrm{i}$ & $0.384675-0.325851 \mathrm{i}$ \\
Exact & $1.133029-0.144984 \mathrm{i}$ & $0.746390-0.436845 \mathrm{i}$ & $0.384675-0.325851 \mathrm{i}$ \\
\hline \hline
\end{tabular}




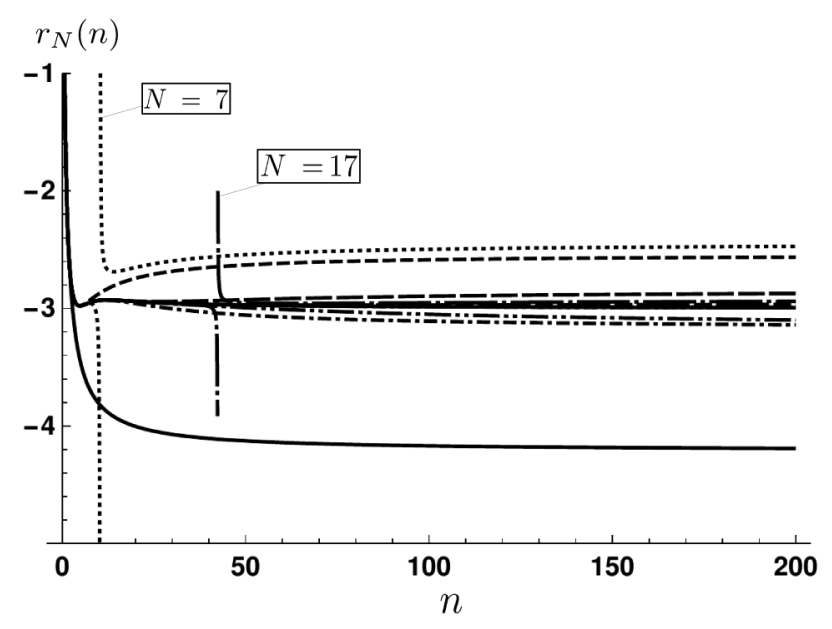

FIG. 3. Hypergeometric ansatz for the quartic anharmonic oscillator. We plot all the rational functions $r_{N}(n)$ for $N \leq 25$ as a function of $n$; these functions approximate the ratio between consecutive coefficients and are used to parametrize hypergeometric approximants in the Borel plane and Meijer- $G$ approximants in the complex- $g$ plane. While we observe good convergence with increased $N$ we note that for $N=7$ and $N=17$, the rational functions have poles for positive $n$, which spoil those two approximants.

are much more accurate than Borel-Padé and that they are competitive with, but much simpler than, VPT.

The expansion coefficients, $e_{n}$, of the ground state energy $E(g) \sim \sum_{n=0}^{\infty} e_{n} g^{n}$ of the quantum quartic anharmonic oscillator with Hamiltonian $H=-d^{2} / d x^{2}+x^{2} / 4+$ $g x^{4} / 4$ are known [9]. For $g>0$ the particle is bound and $E(g)$ is real, but for $g<0$ the particle is unbound and $E(g)$ is complex, mirroring Dyson's collapse scenario [65]: one then expects a branch cut in the complex- $g$ plane, with branch points at $g=0$ and $g=-\infty$. Perturbation theory is thus divergent as the coefficients grow as $e_{n} \sim \Gamma(n+1 / 2)$; the Borel transform then cancels all asymptotic coefficient growth. Taking as input the exact coefficients up to $N=25$ our algorithm yields a table of Meijer- $G$ approximants to
$E(g)$, which we denote $E_{N}(g)$. The asymptotic expansion for the ground state energy is given by

$$
E(g)=\frac{1}{2}+\frac{3}{4} g-\frac{21}{8} g^{2}+\frac{333}{16} g^{3}-\frac{30885}{128} g^{4}+O\left(g^{5}\right),
$$

and the first two Meijer- $G$ approximants read, in numerical form,

$$
\begin{aligned}
& E_{3}(g)=-0.262 G_{2,3}^{3,1}\left(\begin{array}{c}
1,0.758 \\
1,1,-0.270
\end{array} \mid \frac{0.237}{g}\right), \\
& E_{5}(g)=-0.00668 G_{34}^{4,1}\left(\begin{array}{c}
1,0.88+1.18 \mathrm{i}, 0.88-1.18 \mathrm{i} \\
1,1,4.02,-0.321
\end{array} \mid \frac{0.385}{g}\right),
\end{aligned}
$$

When examining the quality of our approximants we note that the $N=7$ and $N=17$ approximants give nonsensical results. This behavior can be attributed to a failure of rational approximations used in the hypergeometric ansatz: looking at the rational functions $r_{N}(n)$ we find that those corresponding to $N=7$ and $N=17$ have a pole for positive $n$ (see Fig. 3). These poles spoil the numerical value of the approximation, by yielding inaccurate approximations to high order coefficient ratios. After acquiring a substantial amount of experience using our approximants, we can say that this behavior is actually typical of rational approximations (Padé approximants also exhibit this kind of behavior), and that it is not at all uncommon to find that a few of the approximants have underlying rational functions that contain poles for positive $n$. As shown in Fig. 3, this minor issue can then easily be checked and identified.

The Meijer- $G$ approximants offer excellent approximations to the ground state energy of the quartic anharmonic oscillator. In Fig. 4 we compare their accuracy with that of VPT and Borel-Padé by plotting the relative error defined
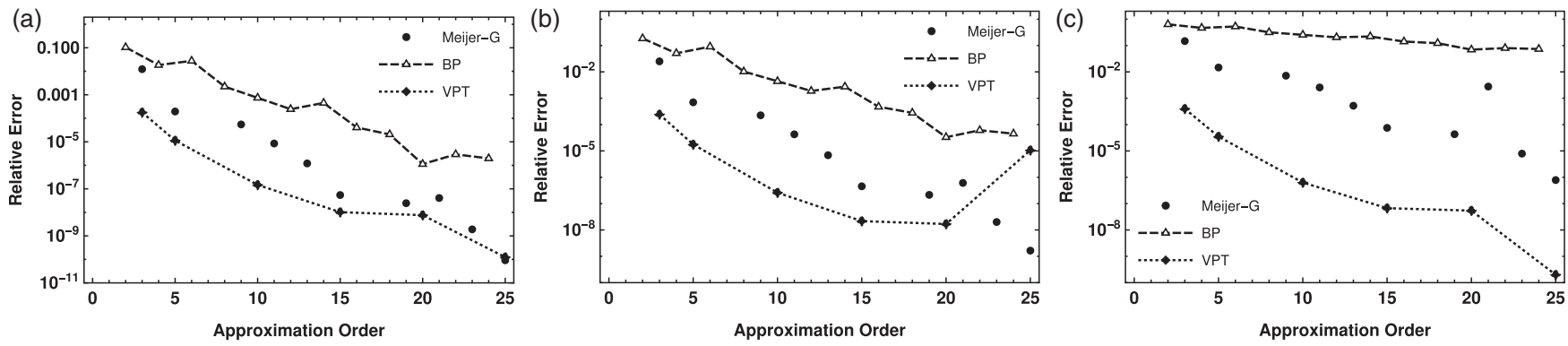

FIG. 4. Accuracy through order plots showing the relative error of Meijer- $G$ approximants (black circles), Borel-Padé (BP; empty triangles with dashed lines), and variational perturbation theory (black diamonds with dotted lines), as a function of the approximation order for different values of $g$ : (a) $g=1$; (b) $g=2$; (c) $g=50$. Note the absent Meijer- $G$ data for $N=7$ and $N=17$ (see text). Overall Meijer- $G$ approximants massively outperform Borel-Padé and are very competitive with the much-more demanding VPT, providing highly accurate results even for strong couplings $(g=50)$ and even surpassing VPT in accuracy for moderate couplings and large orders. 
as $\left|E_{\text {app }}(g) / E_{\text {exact }}(g)-1\right|$ as a function of approximation order. The VPT results have been taken from Table 5.8 in Chap. 5 of Ref. [38]. Clearly the Meijer- $G$ approximants are much more accurate than Borel-Padé approximants and have an accuracy that is typically slightly less than, but clearly competitive with, that of VPT; at large orders $(N \geq 20)$ and moderate couplings $(g=1$ and $g=2)$ our approximants actually produce results that are as accurate or more than the VPT values reported in Ref. [38]. For the strong coupling value $g=50$ our approximants have an accuracy that is midway between Borel-Padé and VPT, numerically:

$$
\begin{aligned}
E_{12 / 12}(g=50) & =2.3157388197, \\
E_{25}(g=50) & =2.4997107287, \\
E_{25, \mathrm{VPT}}(g=50) & =2.4997087731, \\
E(g=50) & =2.4997087726,
\end{aligned}
$$

where $E_{12 / 12}$ is the 24th-order $12 / 12$ Borel-Padé approximation, $E_{25}$ is the 25th-order Meijer- $G$ approximant result, $E_{25, \mathrm{VPT}}$ is the 25th-order VPT result, and $E(g=50)$ refers to the exact numerical value. At such strong couplings Borel-Padé gets only one digit right ( $\sim 10 \%$ error), while Meijer- $G$ gets five digits right, compared to the VPT value reported in Ref. [38] which has nine-digit accuracy. We note in passing that the results from the $N=25$ entry in Table 5.8 of Ref. [38] seem to deviate quite dramatically from the convergence rate one expects from lower order entries. We emphasize that Meijer- $G$ summation is a much simpler approach than VPT, as it can be seen by comparing the algorithm described in Ref. [38] with our algorithm, shown in Sec. II C.

To summarize the results of this section: Meijer- $G$ approximants are remarkably simple but highly accurate approximations to the ground state energy of the quartic anharmonic oscillator. They are much more accurate than Borel-Padé approximants and competitive with much more involved approximations based on VPT.

\section{Anomalous dimension for self-avoiding walks in three dimensions}

In recent work, Kompaniets and Panzer (KP) [10] give the six-loop order $\beta$ function, mass and field anomalous dimension, and critical exponents of the $N$-vector model in 4-2 $\epsilon$ dimensions. Besides adding one extra coefficient to previously computed expansions, their work is an exercise in state-of-the-art practical resummation as well as an excellent compilation of reference results for this important model $[34,66]$. Furthermore KP give full access to their numerical and analytical expansions in their supplementary material. The work of KP then gives us the opportunity to test our summation technique in a realistic physical scenario, where only a handful of coefficients are known and we have no a priori knowledge of the singularity structure in the Borel plane, beyond the large order asymptotics of the expansion coefficients. For simplicity we will consider here the three-dimensional case $(\epsilon=1 / 2)$ and provide only results for $n=0$ (self-avoiding walk case), leaving the application of Meijer- $G$ approximants for other values of $n$ and $\epsilon$ for the future.

To run our algorithm we use the numerical series for the $\beta$ function, the mass anomalous dimension, $\Gamma_{m^{2}}$, and the field anomalous dimension, $\Gamma_{\phi}$ as given by KP. Using the Meijer- $G$ approximations to the $\beta$ function we solve

$$
\beta\left(g^{\star}\right)=0,
$$

which yields the critical coupling, $g^{\star}$. With the critical coupling and the summed-up anomalous dimensions we then compute the critical exponents $\eta$ and $\nu$ as

$$
\begin{gathered}
\eta=2 \Gamma_{\phi}\left(g^{\star}\right), \\
\nu=\frac{1}{2+\Gamma_{m^{2}}\left(g^{\star}\right)},
\end{gathered}
$$

and compare $\eta$ and $\nu$ with the values compiled by KP.

For the $n=0$ case the perturbation expansion for the $\beta$ function is given in numerical form by

$$
\beta(g) \approx-g+2.667 g^{2}-4.667 g^{3}+25.46 g^{4}+O\left(g^{5}\right) .
$$

We start our resummation by building the "helper" function $\beta^{\prime}(g)=1+\beta(g)$, which enables us to obtain oddnumbered approximants of orders $N=3,5$, and 7. Our algorithm then yields the functions

$$
\begin{aligned}
& \beta_{3}^{\prime} \approx 1.185 G_{2,3}^{3,1}\left(\begin{array}{c|c}
1,-1.385 \\
1,1,-1.588
\end{array} \mid \frac{1.147}{g}\right), \\
& \beta_{5}^{\prime} \approx 9.931 \times 10^{10} G_{3,4}^{4,1}\left(\begin{array}{c}
1,15.630,-1.783 \\
1,1,3.217,-1.906
\end{array} \mid \frac{0.220}{g}\right), \\
& \beta_{7}^{\prime} \approx 1.185 G_{4,5}^{5,1}\left(\begin{array}{c}
1,-1.734,-2.791+4.582 \mathrm{i},-2.791-4.582 \mathrm{i} \\
1,1,-0.318+5.571 \mathrm{i},-0.318-5.571 \mathrm{i},-1.884
\end{array} \mid \frac{1.175}{g}\right),
\end{aligned}
$$


and, therefore, the corresponding $\beta_{N}=\beta_{N}^{\prime}-1$. These functions are real for $g>0$, and their examination shows that we have a decently converged result at $N=5$. Then, from solving $\beta_{N}\left(g^{*}\right)=0$ we have three approximations for the critical coupling:

$$
\begin{aligned}
& g_{3}^{\star} \approx 0.6736, \\
& g_{5}^{\star} \approx 0.5339, \\
& g_{7}^{\star} \approx 0.5381 .
\end{aligned}
$$

As our best value we take $g^{\star} \approx g_{7}^{\star}$.

Next we sum the expansion for the mass anomalous dimension. Its perturbation series is given numerically

$$
\begin{aligned}
\Gamma_{m^{2}}(g) \approx & 1-0.667 g+0.556 g^{2}-2.056 g^{3}+10.762 g^{4} \\
& +O\left(g^{5}\right),
\end{aligned}
$$

which is summed using our approximants. For instance, the fifth-order Meijer- $G$ approximant is

$$
\begin{aligned}
& \Gamma_{m^{2}, N=5}(g) \\
& \quad \approx 7159.78 G_{3,4}^{4,1}\left(\begin{array}{c|c}
1,8.64281,-1.17589 & 0.36667 \\
1,1,2.30322,-1.07843 & g
\end{array}\right) .
\end{aligned}
$$

To make the most of the few coefficients we have available we use the once-subtracted Borel-transformed series to obtain even-order approximants. Similar to the quartic anharmonic case of Sec. II B, the rational function $r_{6}(n)$ has a pole at $n \approx 2.5$, which spoils the sixth-order approximant, and therefore we can use only approximants of order $g^{5}$ or lower. Using the summed-up mass anomalous dimension (up to fifth order) and our best value for $g^{\star}$ we compute three approximations to the critical exponent $\nu$, which are

$$
\begin{aligned}
& \nu_{3} \approx 0.5921, \\
& \nu_{4} \approx 0.5865, \\
& \nu_{5} \approx 0.5871 .
\end{aligned}
$$

TABLE II. Comparison of the critical exponents $\eta$ and $\nu$ obtained using the method of Meijer-G approximants with the values reported and compiled by Kompaniets and Panzer (MC data for $\nu$ has been obtained by KP using data from Ref. [66]). Meijer- $G$ approximants yield accurate values for the critical exponents.

\begin{tabular}{lcc}
\hline \hline \multicolumn{3}{c}{ Critical exponents for $\epsilon=1 / 2$ and $n=0$} \\
\hline Method & $\nu$ & $\eta$ \\
\hline Kompaniets-Panzer [10] & 0.5874 & 0.0310 \\
Guida-Zinn-Justin [34] & 0.5875 & 0.0300 \\
This work & 0.5871 & 0.0308 \\
MC result [10,66] & 0.5876 & 0.0310 \\
\hline \hline
\end{tabular}

These values can be compared with accurate Monte Carlo (MC) results that yield $\nu_{\mathrm{MC}}=0.5875970$ as well as with the accurate resummations of Guida and Zinn-Justin [34], which give $\nu_{\mathrm{GZJ}}=0.5875$, and also with those of KP [10], $\nu_{\mathrm{KP}}=0.5874$. Clearly the more complex summation methods used by Guida and Zinn-Justin or KP are more accurate than the much simpler Meijer- $G$ summation which, nevertheless, returns approximations to $\nu$ that are within $1 \%$ of the Monte Carlo result.

We repeat this procedure for the field anomalous dimension, $\Gamma_{\phi}(g)$, calculating the critical exponent $\eta=$ $2 \Gamma_{\phi}\left(g^{\star}\right)$ instead. We take the numerical expansion given by $\mathrm{KP}$ as the starting point

$\Gamma_{\phi}(g) \approx 0.0556 g^{2}-0.0370 g^{3}+0.1929 g^{4}+O\left(g^{5}\right)$.

The resummation is done for the helper function

$\frac{\Gamma_{\phi}(g)}{0.0556 g^{2}} \approx 1-0.6667 g+3.4722 g^{2}-18.1076 g^{3}+O\left(g^{4}\right)$.

Again we use the once-subtracted Borel-transformed series to calculate the fourth-order approximant for the helper function. The resulting approximants are

$$
\begin{aligned}
& \Gamma_{\phi, N=5}(g) \approx 0.0475 g^{2} G_{2,3}^{3,1}\left(\begin{array}{c|c}
1,-0.618 & 0.711 \\
1,1,-0.292618 & \left.\frac{0.2}{g}\right)
\end{array}\right. \\
& \Gamma_{\phi, N=6}(g) \approx 0.0556 g^{2}\left(1-0.534 G_{2,3}^{3,1}\left(\begin{array}{c}
0,-1.056 \\
1,0,-1.081
\end{array} \mid \frac{0.560}{g}\right)\right) .
\end{aligned}
$$

Evaluating these approximants at the critical coupling, we evaluate the critical exponent $\eta$ and obtain

$$
\begin{aligned}
& \eta_{5}=0.03003, \\
& \eta_{6}=0.03083,
\end{aligned}
$$

which are to be compared with the values reported by $\mathrm{KP}, \quad \eta_{K P, \epsilon^{6}} \approx 0.0310$ and $\eta_{\mathrm{KP}, \epsilon^{5}} \approx 0.0314$; Guida and
Zinn-Justin, $\eta_{\mathrm{GZJ}, \epsilon^{5}} \approx 0.0300$; and the accurate Monte Carlo value, $\eta_{\mathrm{MC}} \approx 0.0310$.

The results of this section are summarized in Table II. Clearly the Meijer- $G$ approximants return accurate estimates of both the mass and field anomalous dimensions as well as their critical exponents $\eta$ and $\nu$. Note that KP use as resummation a variational perturbation theory approach, and that Guida and Zinn-Justin use a conformal mapping 
on top of their Borel-Padé method [10,34]. In contrast, the calculations presented here are straightforward to implement, and delivered these results for the most natural choices of helper functions, which were, in fact, the first and only ones we tried. Hence, Meijer- $G$ approximants offer an easy-to-implement alternative to the popular BorelPadé approach.

\section{Remarks}

These findings demonstrate how to easily build accurate Meijer- $G$ approximations to the sum of a Borel-summable divergent series and are confirmed by calculations for various other systems, which will be shown elsewhere. Our approximants should be accurate in cases where the convergence of the Borel-transformed series is limited by a branch point singularity. In this section we have seen how our approximants yield the exact partition function zero-dimensional $\phi^{4}$ theory and very accurate approximations to the ground-state energy of the quartic anharmonic oscillator and anomalous dimensions for self-avoiding walks in three dimensions. In these cases Meijer- $G$ approximants outperform BorelPadé approximants and are competitive with conformal mapping and variational perturbation theory techniques. In the next section we investigate the applicability of Meijer- $G$ approximants to problems where perturbation theory is not Borel summable.

\section{SUMMATION OF RESURGENT TRANSSERIES}

In many cases perturbation expansions are not Borel summable [25]. In such cases one needs to sum a resurgent transseries. In the examples below it is shown that Meijer- $G$ approximants can also be used to provide economical and accurate approximations to the "sum" of such expansions. In particular, in Sec. IV A we will consider the summation of the partition function in $\phi^{4}$ theory with degenerate minima [67], as well as that of a self-interacting QFT model in zero dimensions [29] in Sec. IV B. These examples are often utilized for benchmarking new summation techniques, since they contain highly divergent series that are not Borel summable. It will be shown that, remarkably, these cases constitute a best-case scenario for the application of the Meijer- $G$ summation technique developed above. As we saw in Sec. III A, this is so because the Meijer- $G$ approximants converge at order $N=5$. This means that by applying the Meijer- $G$ summation procedure we arrived at a closed-form analytic expression. In other words, these partition functions belong to the space of functions reproducible by our summation technique, and thus they are easily summable by means of Meijer- $G$ approximants. The reason for this is that in these cases the hypergeometric ansatz turns out to be exact, and the Borel-transformed series sums exactly to a hypergeometric function of the form ${ }_{3} F_{2}\left(1, h_{1}, h_{2} ; h_{3}, h_{4}, h_{5} \tau\right)$ [or, equivalently, to a hypergeometric function of the form $\left.{ }_{2} F_{1}\left(h_{1}, h_{2}, h_{3}, h_{4} \tau\right)\right]$. Therefore, in Sec. III D we complement our study by considering the summation of the transseries expansion for the double-well potential in quantum mechanics, including the one- and two-instanton contributions [28], which constitutes a more challenging example since the approximants exhibit highly nontrivial convergence properties.

\section{A. Degenerate vacua}

Marucho [67] considered a partition function of the form

$$
Z(g)=\frac{1}{\sqrt{2 \pi}} \int_{-\infty}^{\infty} e^{-\phi^{2}(1-\sqrt{g} \phi)^{2} / 2} d \phi, \quad g>0 .
$$

The term $\phi^{2} / 2$ in the exponent may be regarded as a free-field $(g=0)$, and a traditional perturbative approach, such as diagrammatics, results in an asymptotic expansion in powers of $g$. The first few terms of this expansion are

$$
Z(g) \sim 1+6 g+210 g^{2}+13860 g^{3}+\cdots,
$$

and its general term can be found in the paper in Ref. [67]. Hence we are once again in a situation where we happen to know all of the coefficients. We stress that this only happens in toy models and expansion coefficients are rarely known at large orders. With these coefficients we can easily run our algorithm and find the odd order Meijer$G$ approximants, which read

$$
\begin{aligned}
& Z_{B, 3}(g)=-\frac{0.00733}{g} G_{2,3}^{3,1}\left(\begin{array}{c}
0,0.286 \\
-0.761,0,0
\end{array} \mid-\frac{0.0310}{g}\right), \\
& Z_{B, 5}(g)=-\frac{0.00701}{g} G_{3,4}^{4,1}\left(\begin{array}{c}
0,0,0 \\
-0.25,-0.75,0,0
\end{array} \mid-\frac{0.0312}{g}\right), \\
& Z_{B, 7}(g)=-\frac{0.00701}{g} G_{4,3}^{5,1}\left(\begin{array}{c}
0,0,0,0 \\
-0.25,-0.75,0,0,0
\end{array} \mid-\frac{0.0312}{g}\right),
\end{aligned}
$$

These Meijer- $G$ approximants to the Borel sum are fully converged at order $N=5$ and larger; by increasing the order of the approximants we get the same function again and again: $Z_{B, 5}=Z_{B, 7}=\cdots$. This means that we have found the exact Borel sum by means of our approach; the Borel sum of $Z(g)$ belongs to the space of reproducible functions associated with Borel-hypergeometric resummation. The converged approximants agree with the exact Borel sum [67] given by

$$
Z_{B}(g) \approx \frac{\mathrm{i} 0.1 e^{-0.0156 / g}}{\sqrt{x}} K_{-1 / 4}\left(-\frac{0.0156}{g}\right) .
$$



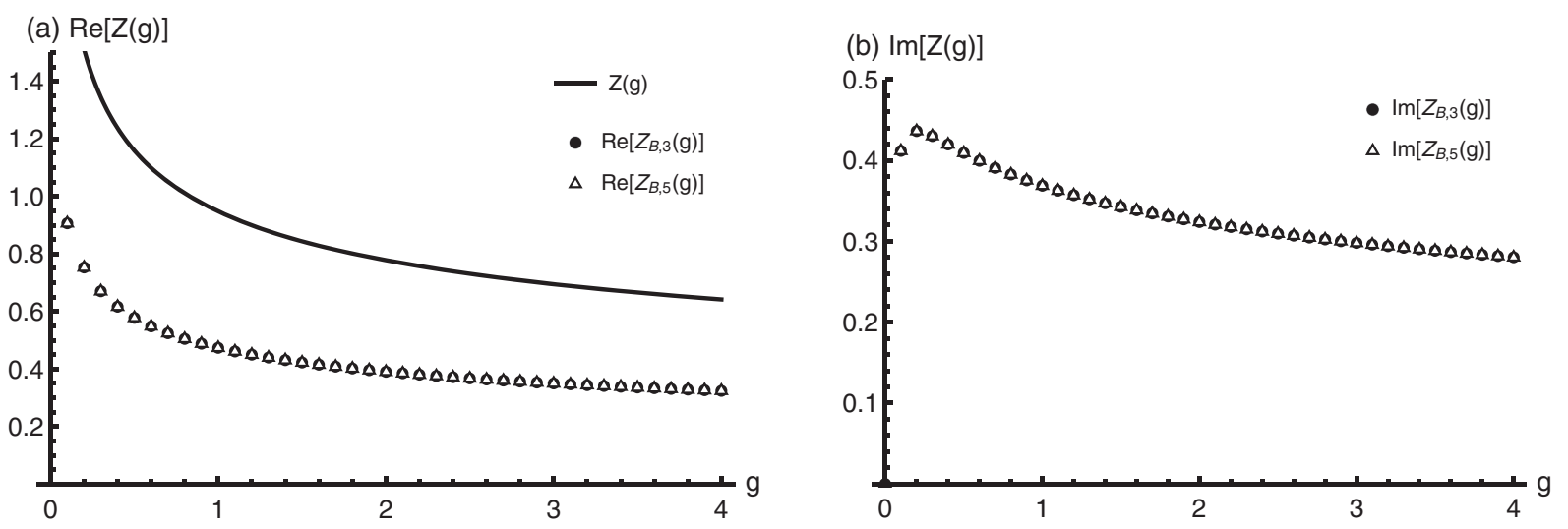

FIG. 5. Partition function, $Z(g)$, for $\phi^{4}$ theory with degenerate minima. (a) The real part of the exact $Z(g)$ (solid curve) is compared with the real part of the third- $\left[Z_{B, 3}(g)\right.$, filled circles $]$ and fifth-order $\left[Z_{B, 5}(g)\right.$, empty triangles] Meijer- $G$ approximants to the Borel sum of $Z(g)$. The Meijer- $G$ approximants are well converged for the range of values of $g$ shown and are not clearly distinguishable on the scale of the plot. The fifth-order approximant is converged to the exact Borel sum given by Marucho [67]. The real part of the Borel sum underestimates $Z(g)$ by a factor of $2, \operatorname{Re}\left[Z_{B, 5}(g)\right]=Z(g) / 2$. (b) Imaginary parts of the Meijer- $G$ approximants are not zero, while $Z(g)$ is manifestly real, $\operatorname{Im}\left[Z_{B, 5}(g)\right] \neq 0$ while $\operatorname{Im}[Z(g)]=0$. Perturbation theory for $\phi^{4}$ with degenerate minima is not Borel summable.

Our approximants actually find the exact Borel sum for this problem with just a few orders. In contrast, Borel-Padé would have required very high order coefficients to produce accurate results up to modest values of the coupling. Unfortunately-for the present problemhaving very accurate approximations to the Borel sum turns out to be insufficient to accurately approximate $Z(g)$. The problem is, of course, that $Z(g)$ is not Borel summable. This is clearly seen in Fig. 5 which shows the real and imaginary parts of $Z_{B, 3}(g)$ and $Z_{B, 5}(g)$ together with the exact $Z(g)$. The Borel sum fails to accurately represent $Z(g)$; in Fig. 5(a) we see that the real part of the Borel sum is off by a constant factor of 2; in Fig. 5(b) we see that the Borel sum possesses an imaginary part, while $Z(g)$ is manifestly real. Note that the $N=3$ approximant is nearly converged while the $N=5$ approximant is fully converged to the exact value of the Borel sum, given in Ref. [67].

When performing a Borel summation it is not uncommon to obtain imaginary parts. While in some cases it is rather straightforward to give them a physical meaning, in the present case it is not so clear and furthermore such physical interpretation is not our objective-which is to accurately approximate $Z(g)$. This imaginary part is called nonperturbative ambiguity. It is ambiguous because it changes sign depending on whether one evaluates $Z_{B}(g+$ $\mathrm{i} \epsilon)$ or $Z_{B}(g-\mathrm{i} \epsilon)$, i.e., $\operatorname{Im}\left[Z_{B}(g+\mathrm{i} \epsilon)\right]=-\operatorname{Im}\left[Z_{B}(g-\mathrm{i} \epsilon)\right]$. Non-Borel summability for this problem is explained in Ref. [67]. For completeness we give our version here (see Fig. 6). For very small but nonzero couplings the integrand in $Z(g)$ contains contributions from two identical Gaussians, each of which sits on a different saddle, one at $x=0$ and the other at $x=1 / \sqrt{g}$. In the literature the former is referred to as the "perturbative saddle" while the latter is known as the "nonperturbative saddle." If the area under each Gaussian is $A$, then $Z\left(g \rightarrow 0^{+}\right)=2 A$. When doing perturbation theory around $g=0$ one is effectively taking into account contributions only from the perturbative saddle and thus the perturbative estimate will be $Z\left(g \rightarrow 0^{+}\right)=A$, which is wrong by a factor of 2 . The Meijer- $G$ approximants succeed in summing up the perturbation expansion around $g=0$ from only a few terms, but that is not enough. The Borel sum misses the contribution from the second Gaussian entirely and thus underestimates the real part of $Z(g)$ by a factor of 2 . It is then clear that $Z(g)$ has an essential singularity at $g=0$. Perturbation theory is not sufficient to accurately evaluate $Z(g)$ even in the limit $g \rightarrow 0$.

To obtain an accurate estimate of $Z(g)$ we need to upgrade the perturbation expansion to a resurgent transseries which, for the present problem, is of the form

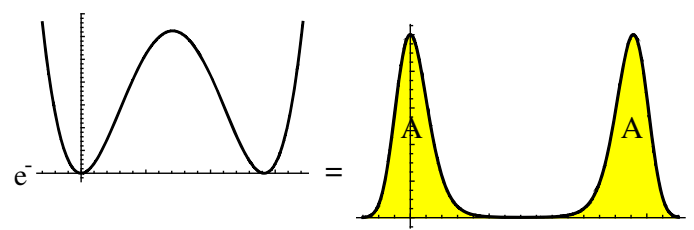

FIG. 6. Summability problem in $\phi^{4}$ theory with degenerate minima. Exponentiating a symmetric double well with $g=0.005$ yields two equal-area Gaussians, each centered on its respective well. The left well is called perturbative saddle, and the right well is called nonperturbative saddle. For any $g>0$, no matter how small, $Z(g)$ is the total area under the Gaussians. Borel summation of perturbation theory yields exactly $Z(g) / 2$ plus an imaginary part called the nonperturbative ambiguity, which is exponentially suppressed as $g \rightarrow 0$. The Borel sum of the standard divergent perturbation theory of $Z(g)$ accounts only for contributions coming from the perturbative saddle. 


$$
\begin{aligned}
Z(g)= & \pm \mathrm{i} \sqrt{g} e^{-\frac{1}{32 g}} F_{0}(1 / 4,3 / 4,-32 g) \\
& +2{ }_{2} F_{0}(1 / 4,3 / 4,32 g) \\
= & \pm \mathrm{i} \sqrt{g} e^{-\frac{1}{32 g}}\left(1-6 g+210 g^{2}-\cdots\right) \\
& +2\left(1+6 g+210 g^{2}+\cdots\right),
\end{aligned}
$$

where the ${ }_{2} F_{0}$ factors are divergent hypergeometric series and the upper sign is for $\operatorname{Im} g>0$, while the lower sign is for $\operatorname{Im} g<0$. This transseries contains two divergent series that need to be summed. The so-called Borel-Padé-Écalle method uses the Borel-Padé technique to sum each of these divergent series [17-20,22,29]. Here we use instead Meijer- $G$ approximants to sum each of the divergent series that appears in the transseries expansion. Such an approach can then be described as the Borel-hypergeometric-Écalle summation method. Meijer- $G$ approximants are extremely well suited to sum this expansion exactly since-as discussed in Sec. II-they are regularizing analytic continuations of the divergent hypergeometric series ${ }_{n+2} F_{n}$. Therefore the exact Borel sum of each of the two divergent hypergeometric functions that enter Eq. (55) can be obtained directly from Meijer- $G$ approximants of order $N \geq 5$. Defining

$$
\begin{gathered}
Z_{1}(g) \equiv \pm \mathrm{i} \sqrt{g} e^{-\frac{1}{32 g}} F_{2}(1 / 4,3 / 4,-32 g), \\
Z_{2}(g) \equiv 2_{2} F_{0}(1 / 4,3 / 4,32 g),
\end{gathered}
$$

we can find their respective $N$ th-order Meijer- $G$ approximations to their Borel sums, denoted $Z_{B, 1, N}$ and $Z_{B, 2, N}$. The third-order approximants are

$$
\begin{aligned}
& Z_{B, 1,3}(g)= \pm e^{-\frac{1}{32 g}} \frac{\mathrm{i} 0.00733}{\sqrt{g}} G_{2,3}^{3,1}\left(\begin{array}{c}
0,0.286 \\
-0.761,0,0
\end{array} \mid \frac{0.0310}{g}\right), \\
& Z_{B, 2,3}(g)=-2 \frac{0.00733}{g} G_{2,3}^{3,1}\left(\begin{array}{c}
0,0.286 \\
-0.761,0,0
\end{array} \mid-\frac{0.0310}{g}\right),
\end{aligned}
$$

and the third-order approximation to the full transseries is

$$
Z(g)=Z_{B, 1,3}(g)+Z_{B, 2,3}(g)+O\left(g^{4}\right),
$$

while the fifth-order approximant is converged to the exact value. The fifth-order approximation to the transseries actually reconstructs $Z(g)$ exactly:

$$
Z(g)=Z_{B, 1,5}(g)+Z_{B, 2,5}(g) .
$$

Hence we use Meijer- $G$ functions to sum up the ${ }_{2} F_{0}$ divergent hypergeometric series that appears in the transseries given by Eq. (55). In Fig. 7 we compare the exact $Z(g)$ with the results of third- and fifth-order Borel-hypergeometric-Écalle summation. In Fig. 7(a) we see that third-order and fifth-order Meijer- $G$ approximants are excellent approximations to the exact $\operatorname{Re} Z(g)$; the factor of 2 discrepancy has
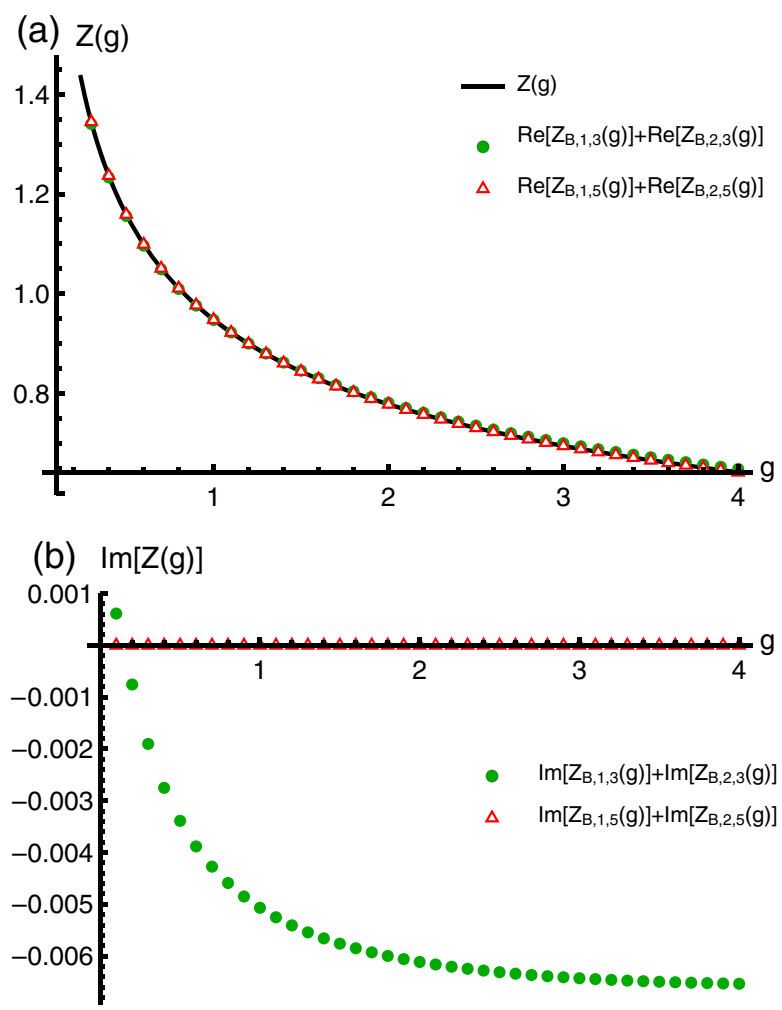

FIG. 7. Transseries summation by Meijer- $G$ approximants for $\phi^{4}$ theory with degenerate minima. (a) Exact real part of $Z(g)$ versus third- and fifth-order summed-up transseries. (b) Imaginary part of $Z(g)$ as computed by third-order and fifth-order Meijer- $G$ approximants; the third-order Meijer- $G$ approximants nearly cancel the nonperturbtaive ambiguity [compare with Fig. 5(b)]; the fifth-order approximants are exact and thus have zero imaginary part.

been removed by proper transseries summation. In Fig. 7(b) we see that the nonperturbative ambiguity has also been canceled; while for third-order Meijer- $G$ approximants this cancellation is not complete as the size of the ambiguity has been reduced by 2 orders of magnitude, as can be seen by comparing Fig. 7(b) with Fig. 5(b). The cancellation is exact for fifth-order Meijer- $G$ approximants that sum the transseries exactly. Table III shows both the nonperturbative ambiguity and the missing factor of 2 in the Meijer- $G$ approximants to the Borel sum of $Z(g)\left(Z_{B, 3}\right.$ and $\left.Z_{B, 5}\right)$ for selected values of $g$ (ranging from intermediate to very strong couplings). When the Borel-hypergeometric-Écalle summation is adopted, the ambiguity is removed and the factor of 2 is restored. The third-order approximant, while not exact, is remarkably accurate.

Using the integral considered by Marucho [67] we have illustrated the application of Meijer- $G$ approximants to transseries summation. Such an approach can be thought of as Borel-hypergeometric-Écalle summation, as opposed to Borel-Padé-Écalle and consists in using Meijer- $G$ approximants (or hypergeometric approximants on the Borel plane; or rational approximations for the ratio between consecutive coefficients) to sum the divergent series that contributes to the 
TABLE III. $Z(g)$ for zero-dimensional $\phi^{4}$ theory with degenerate minima evaluated for $g=1, g=10$, and $g=100$ using the thirdand fifth-order Meijer- $G$ approximants to the Borel sum, $Z_{B, 3}(g)$ and $Z_{B, 5}(g)$, as well as the third- and fifth-order Meijer- $G$ approximations to the resurgent transseries, $Z_{B, 1,3}(g)+Z_{B, 2,3}(g)$ and $Z_{B, 1,5}(g)+Z_{B, 2,5}(g)$. We also include the exact result obtained by evaluating $Z(g)$ numerically in Eq. (52). The approximations to the Borel sum fail to reproduce both the real part and the imaginary part of the exact result. In contrast the Meijer- $G$ resummation of the resurgent transseries for this problem gives a better account of the exact $Z(g)$ : the third-order Meijer- $G$ approximant is in excellent agreement with the exact result, nearly canceling the nonperturbative ambiguity; the fifth-order Meijer- $G$ approximant is exact.

\begin{tabular}{lccc}
\hline \hline Approximant & $g=1$ & $g=10$ & $g=100$ \\
\hline$Z_{B, 3}(g)$ & $0.473794+0.368724 \mathrm{i}$ & $0.255694+0.228610 \mathrm{i}$ & $0.144490+0.133539 \mathrm{i}$ \\
$Z_{B, 5}(g)$ & $0.473980+0.372796 \mathrm{i}$ & $0.250564+0.232317 \mathrm{i}$ & $0.137588+0.134338 \mathrm{i}$ \\
$Z_{B, 1,3}(g)+Z_{B, 2,3}(g)$ & $0.947587-0.010133 \mathrm{i}$ & $0.511388-0.012758 \mathrm{i}$ & $0.288979-0.009096 \mathrm{i}$ \\
$Z_{B, 1,5}(g)+Z_{B, 2,5}(g)$ & 0.947959 & 0.501129 & 0.275175 \\
Exact & 0.947959 & 0.501129 & 0.275175 \\
\hline \hline
\end{tabular}

resurgent transseries. In the present case we found that the transseries can be summed exactly with fifth-order Meijer- $G$ approximants. While not exact, the third-order approximation provides excellent approximations to $Z(g)$ and removes most of the nonperturbative ambiguity.

\section{B. Self-interacting QFT}

Reference [29] considers the following partition function of a " $(0+0)$-dimensional self-interacting QFT"

$$
Z(g)=\frac{1}{\sqrt{2 \pi g}} \int_{-\pi / 2}^{\pi / 2} e^{\frac{\sin (\phi)^{2}}{2 g}} d \phi=\frac{\pi}{\sqrt{g}} e^{-\frac{1}{4 g}} I_{0}\left(\frac{1}{4 g}\right),
$$

where $I_{0}(x)$ is a modified Bessel function of the first kind. $Z(g)$ has the asymptotic expansion

$$
Z(g) \sim 1+\frac{g}{2}+\frac{9 g^{2}}{8}+\frac{75 g^{3}}{16}+\frac{3675 g^{4}}{128}+\cdots .
$$

Using the techniques put forward in Sec. II we once again obtain a convergent set of Meijer- $G$ approximations to the Borel sum of this series. The converged Meijer- $G$ approximant to the Borel sum is of fifth order and reads

$$
\begin{aligned}
Z_{B, 5}(g) & =-\frac{1}{4 \pi g} G_{3,4}^{4,1}\left(\begin{array}{c}
0,0,0 \\
-1 / 2,-1 / 2,0,0
\end{array} \mid-\frac{1}{4 g}\right) \\
& =-\frac{1}{2 \pi g} e^{-\frac{1}{4 g}} K_{0}\left(-\frac{1}{4 g}\right)
\end{aligned}
$$

As in the previous example, increasing the order of the approximant yields the same Meijer- $G$ function. The converged approximants can be compared with the very accurate, but not exact, third-order Meijer- $G$ approximant

$Z_{B, 3}(g)=-\frac{26}{53 g} \frac{\Gamma\left(\frac{19}{13}\right)}{\Gamma\left(\frac{19}{53}\right)} G_{2,3}^{3,1}\left(\begin{array}{c|c}0,6 / 13 & 26 \\ -34 / 53,0,0 & -\frac{2}{53 g}\end{array}\right)$.

Evaluating $Z(g)$ and $Z_{B, 5}(g)$ numerically for selected values of $g$ we see that

$$
\operatorname{Re}\left[Z_{B, 5}(g)\right]=\operatorname{Re}[Z(g)]
$$

but

$$
\operatorname{Im}\left[Z_{B, 5}(g)\right] \neq 0
$$

while

$$
\operatorname{Im}[Z(g)]=0
$$

Thus we find the nonperturbative ambiguity once again. For example, evaluating the third- and fifth-order approximants at $g=1$ we obtain

$$
\begin{aligned}
Z_{B, 3}(g=1 \pm \mathrm{i} \epsilon)= & 0.9903122408877890894 \ldots \\
& \pm \mathrm{i} 0.4813082375368570801 \ldots, \\
Z_{B, 5}(g=1 \pm \mathrm{i} \epsilon)= & 0.9913929921688975613 \ldots \\
& \pm \mathrm{i} 0.4789408454106600542 \ldots
\end{aligned}
$$

while

$$
Z(g=1)=0.9913929921688975613 \ldots
$$

Repeating at the very large value of $g=100$ we find

$$
\begin{aligned}
Z_{B, 3}(g=100 \pm \mathrm{i} \epsilon)= & 0.13677671640883210679 \ldots \\
& \pm \mathrm{i} 0.23483780883795517888 \ldots \\
Z_{B, 5}(g=100 \pm \mathrm{i} \epsilon)= & 0.12501867187315494524 \ldots \\
& \pm \mathrm{i} 0.24304192933460168829 \ldots
\end{aligned}
$$

while

$$
Z(g=100)=0.12501867187315494524 \ldots
$$

This comparison shows that the third-order Meijer- $G$ approximants are excellent approximations to the exact Borel sum, and that fifth-order Meijer- $G$ approximants reproduce the exact Borel sum. Finally it is clear that the 
series is not Borel summable: using the Borel sum to estimate the value of $Z(g)$ results in a fictitious imaginary part with no clear physical interpretation. The Borel sum fails to reproduce the analytic structure of $Z(g)$ on the complexified $g$ plane.

Cherman et al. [29] provide a very clear explanation of the appearance of the nonperturbative ambiguity in this problem, which echoes the discussion given above for the case of $\phi^{4}$ theory with degenerate vacua. In the present case, we have also an action $\frac{\sin (\phi)^{2}}{2 g}$ with a perturbative saddle at $\phi=0$, with action $S_{1}=0$, as well as a nonperturbative saddle at $\phi=\pi / 2$ where the action evaluates to $1 /(4 g)\left[S_{2}=1 /(4 g)\right]$. To properly evaluate $Z(g)$ we thus need to sum a transseries expansion of the form

$Z\left(g, \sigma_{0}, \sigma_{1}\right) \sim \sigma_{0} e^{S_{0}} \sum_{n=0}^{\infty} c_{n, 0} g^{n}+\sigma_{1} e^{S_{1}} \sum_{n=0}^{\infty} c_{n, 1} g^{n}$,

where $c_{n, 0}\left(c_{n, 1}\right)$ is the $n$ th-order expansion coefficient around the perturbative (nonperturbative) saddle, and $\sigma_{0}$ and $\sigma_{1}$ are the corresponding transseries parameters. The transseries parameters have been found to be given by $\sigma_{0}=1$ and $\sigma_{1}=-\mathrm{i}$, for

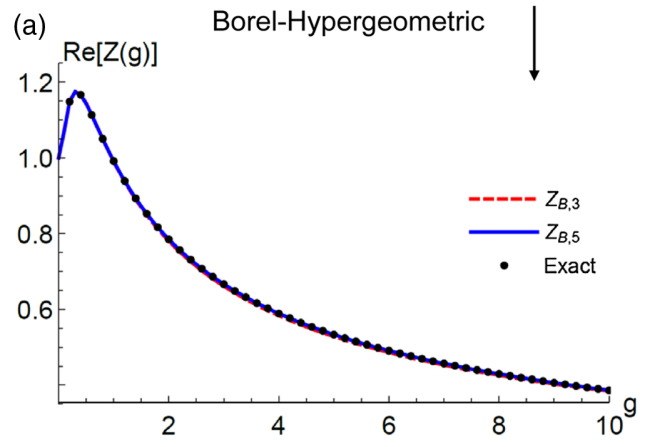

(b)

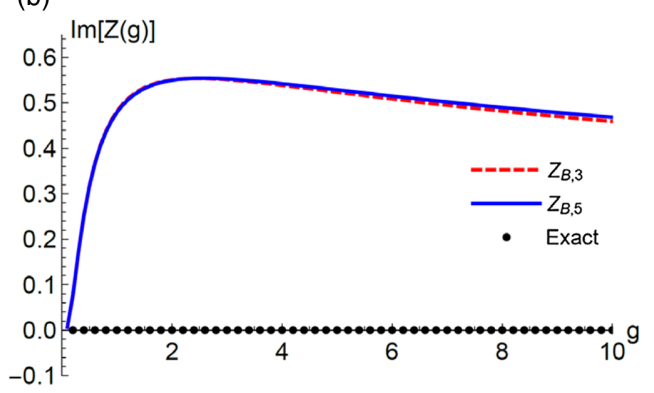

$\operatorname{Im} g>0$ and $\sigma_{1}=\mathrm{i}$, for $\operatorname{Im} g<0$. The divergent series in the transseries expansion can both be summed exactly by Borelhypergeometric summation; the Borel-transformed series are both hypergeometric series

$$
\sum_{n=0}^{\infty} \frac{c_{n, 0}}{n !} \tau^{n} \sim_{2} F_{1}\left(\frac{1}{2}, \frac{1}{2}, 1 ; 2 \tau\right)
$$

and

$$
\sum_{n=0}^{\infty} \frac{c_{n, 1}}{n !} \tau^{n} \sim_{2} F_{1}\left(\frac{1}{2}, \frac{1}{2}, 1 ;-2 \tau\right)
$$

and thus belong to the space of functions that can be reconstructed by our technique. In particular, third-order Meijer- $G$ approximants cannot be exact, as the hypergeometric approximants in the Borel plane are of the form ${ }_{2} F_{1}\left(1, h_{1}, h_{2}, h_{3} \tau\right)$ rather than ${ }_{2} F_{1}\left(1 / 2, h_{1}, h_{2}, h_{3} \tau\right)$. But fifth-order Meijer- $G$ approximants can be exact since

$$
{ }_{2} F_{1}\left(\frac{1}{2}, \frac{1}{2}, 1 ; 2 \tau\right)={ }_{3} F_{2}\left(\frac{1}{2}, \frac{1}{2}, 1,1,1 ; 2 \tau\right),
$$
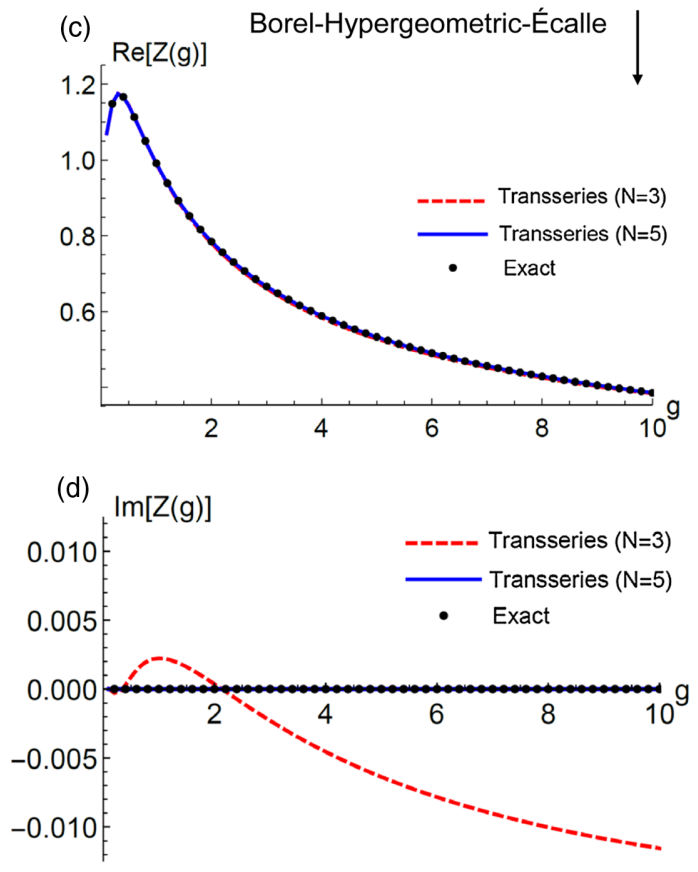

FIG. 8. $Z(g)$ for self-interacting zero-dimensional QFT as a function of $g$ calculated using Meijer- $G$ approximants to the Borel sum (Borel-hypergeometric summation; left panels) and to the Borel-Écalle sum (Borel-hypergeometric-Écalle sum, right panels), and compared with the exact result (dots). (a) Real part of the Borel sum calculated using Meijer- $G$ approximants of order three (dashed curves) and five (solid curves), compared to the exact result; the approximants return excellent approximations to $\operatorname{Re}[Z(g)]$. (b) As in (a), but for $\operatorname{Im}[Z(g)]$; while the Meijer- $G$ approximants are very nearly converged for the range of values of $g$ shown, they fail to reproduce the exact value, $\operatorname{Im}[Z(g)]=0$. (c) As in (a), but this time we use Meijer- $G$ approximants of third order (dashed curves) and fifth order (solid curves) to approximate the Borel-Écalle sum, obtaining once again excellent agreement with the exact $\operatorname{Re}[Z(g)]$. (d) As in (c) but for $\operatorname{Im}[Z(g)]$; the combination of Meijer- $G$ approximants with the BorelÉcalle approach results in a nearly complete cancellation of the nonperturbative ambiguity at third order-the cancellation is complete for $N=5$. 
and therefore the Borel transformed series can be summed up exactly with fifth-order Meijer- $G$ approximants. In Figs. 8(a) and 8(b) we compare Meijer- $G$ (or Borel-hypergeometric) approximants with the exact $Z(g)$. In Fig. 8(a) we show the real part of $Z(g)$ : the third-order approximant already gives an excellent approximation to $\operatorname{Re}[Z(g)]$ while the fifth-order approximant reproduces its exact value. In Fig. 8(b) we see that both approximants have a nonperturbative ambiguity, which is again well approximated by the third-order approximant. The nonperturbative ambiguity can be removed by the transseries summation by Meijer$G$ approximants, as shown in Figs. 8(c) and 8(d). The real part of $Z(g)$, shown in Fig 8(c), is unaffected, but the nonperturbative ambiguity has been effectively removed. Comparing Fig. 8(b) with Fig. 8(d) we see that third-order transseries summation dramatically reduced the size of the nonperturbative ambiguity.

For testing and pedagogical purposes we have chosen the problem of approximating non-Borel-summable partition functions in zero-dimensional QFT, using Meijer- $G$ functions to approximate the Borel sums of the multiple divergent series that appear in a resurgent transseries. Such an approach can be described as the Borel-hypergeometricÉcalle summation method and for the previous two examples delivers the exact answer at the fifth-order level, completely removing the nonperturbative ambiguity. As a final application we discuss the computation of one- and two-instanton corrections in the quantum mechanical doublewell problem.

\section{Summed-up one- and two-instanton contributions in the double-well problem}

A more challenging case is provided by multi-instanton resummation in the quantum-mechanical double-well problem. This is a simple quantum-mechanical problem, but one that is not tractable by standard perturbation theory or by any resummation of perturbative data; a successful resummation requires the construction of a transseries (or multi-instanton expansion) [28], and here we use Meijer-G approximants in an attempt to sum such expansion.
The double-well potential is given by

$$
V(x)=\frac{1}{2} x^{2}(1-\sqrt{g} x)^{2} .
$$

For $g=0$ we have a harmonic oscillator with energy $E_{0}=1 / 2$. However, as we increase $g$, the ground state splits into two different states with opposite parity. The eigenvalue of the lower energy state (first excited state) is denoted $E_{+}\left(E_{-}\right)$. The energy gap between these two states is a nonperturbative and not-Borel-summable quantity-it is exponentially small in the limit $g \rightarrow 0$ and hence not detectable by standard perturbation theory. This is shown in Fig. 9, where $E_{ \pm}$are shown as functions of $g$ together with Meijer- $G$ approximations $(N=3,4,5)$ to the Borel sum. Clearly the Meijer- $G$ approximants succeed at rapidly converging the Borel sum, but the Borel sum is insufficient to describe the nonperturbative energy gap between $E_{+}$and $E_{-}$. The multi-instanton expansion (or resurgent transseries) proposed by Zinn-Justin and Jenstchura [28] can be used to estimate $E_{ \pm}(g)$. Defining

$$
\begin{gathered}
\xi=\frac{1}{\sqrt{\pi g}} e^{-\frac{1}{4 g}}, \\
\chi=\ln \left(-\frac{2}{g}\right), \\
\epsilon= \pm,
\end{gathered}
$$

and neglecting multi-instanton contributions beyond the two-instanton order, $E_{\epsilon}(g)$ is approximated as

$$
E_{\epsilon}(g) \approx E_{0}(g)+E_{\epsilon}^{(1)}(g)+E_{\epsilon}^{(2)}(g),
$$

where $E_{0}(g)$ is the perturbation expansion, $E_{\epsilon}^{(1)}(g)$ is the one-instanton correction and $E_{\epsilon}^{(2)}(g)$ is the two-instanton correction. These are given by

$$
E_{0}(g)=\frac{1}{2}-g-\frac{9}{2} g^{2}-\frac{89}{2} g^{3}-\frac{5013}{8} g^{4}+O\left(g^{5}\right),
$$

$$
\begin{aligned}
E_{\epsilon}^{(2)}(g)= & \xi^{2}(g) \chi(g)\left(1-\frac{53}{6} g-\frac{1277}{72} g^{2}-\frac{336437}{1296} g^{3}+O\left(g^{4}\right)\right) \\
& +\xi^{2}(g)\left(\gamma-\left(\frac{23}{2}+\frac{53}{6} \gamma\right) g-\left(-\frac{13}{12}+\frac{1277}{72} \gamma\right) g^{2}-\left(\frac{45941}{144}+\frac{336437}{1296} \gamma\right) g^{3}+O\left(g^{4}\right)\right),
\end{aligned}
$$




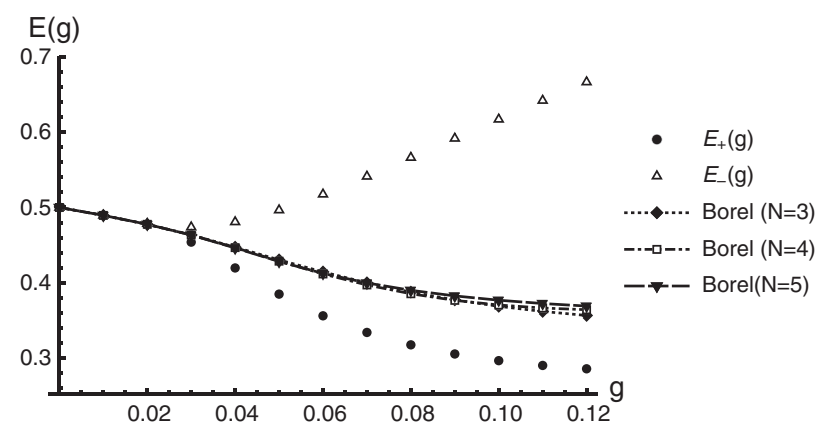

FIG. 9. The perturbation for the quantum-mechanical doublewell problem is not Borel summable. For $g=0$ the ground state has twofold degeneracy, corresponding to states with positive and negative parity $\left(E_{+}\right.$and $E_{-}$; filled circles and empty triangles, respectively). For $g \neq 0$ this degeneracy is broken and $E_{+} \neq E_{-}$. The energy gap that appears between the ground and first excited state is a truly nonperturbative effect and cannot be accounted for by Borel summation (see legend). The Borel sums have been performed by Meijer- $G$ approximants of orders three to five, and appear well converged for the range of values of $g$ shown.

where $\gamma$ is Euler's constant. We can see that this resurgent expansion consists of the standard perturbation expansion plus corrections; each of these corrections contains one or more divergent expansion in powers of $g$, which are multiplied by nonanalytic terms such as $\xi(g), \xi^{2}(g)$, and $\xi(g) \chi(g)$. For instance, the perturbation expansion in Eq. (78) is a divergent series. Similarly for $E_{\epsilon}^{(1)}(g)$ in Eq. (79), the series that multiplies $-\epsilon \xi(g)$ is also divergent. Generalized ad infinitum a resurgent multi-instanton expansion can be described as a series in powers of $\xi(g)$ and $\chi(g)$ where the "expansion coefficients" are themselves divergent series that need to be resummed. The zeroth order of such expansion is the traditional perturbation theory. Higher order terms contain powers of the form $\xi^{n}(g) \chi^{m}(g)$ multiplying a divergent series.

In the present case contributions at odd instanton orders are parity dependent: they have opposite signs depending on the parity. In contrast, even-order multi-instanton contributions are parity independent [28]. Thus the oneinstanton correction opens the gap between $E_{+}$and $E_{-}$as these states have opposite parity; the one-instanton correction lowers the energy of + state, increasing the energy of state, and therefore breaks the degeneracy observed at $g=0$. In contrast, the two-instanton contribution tends to increase the energy of both + and - states.

We will attempt to sum the resurgent transseries given by Eq. (77) by replacing each divergent series that contributes to $E_{0}(g), E_{\epsilon}^{(1)}(g)$, and $E_{\epsilon}^{(2)}(g)$ in Eqs. (78)(80) by their respective Borel sums. The Borel sums will be calculated using Meijer- $G$ approximants, including powers in $g$ of order seven or lower. Because the convergence of the approximants turned out to be slower than in the previous examples, here we calculate also the even-ordered approximants by starting the algorithm described above from the once-subtracted series. For clarity we will show only results obtained by seventhorder Meijer- $G$ approximants, describing briefly the apparent convergence of the approximants when necessary. We denote the $N$ th-order $n$-instanton approximations to $E_{\epsilon}$ as $E_{\epsilon, n, N}$. As mentioned above, the $n$-instanton approximations are replaced by Meijer- $G$ approximants of order $N \leq 7$; i.e., we perform a Borel-hypergeometricÉcalle summation. For instance, the third-order oneinstanton approximation is roughly given by

$$
\begin{aligned}
E_{ \pm, 1,3}(g) \approx & \frac{0.0288}{g} G_{2,3}^{3,1}\left(\begin{array}{c}
0,-0.347 \\
-1.26,0,0
\end{array} \mid-\frac{0.199}{g}\right) \\
& \mp \frac{0.0222}{g} \xi(g) G_{2,3}^{3,1}\left(\begin{array}{c}
0,-0.255 \\
-1.58,0,0
\end{array} \mid-\frac{0.131}{g}\right) .
\end{aligned}
$$

In Fig. 9 we show how the coupling breaks the degeneracy between + and - states. Numerically exact values are compared with Meijer- $G$ approximants to the Borel sum (zero-instanton) $E_{ \pm, 0, N}$ with $N=3-5$. The approximants appear to be well converged for the range of values of $g$ shown. The Borel sum does not account for the splitting between + and - states. To account for this nonperturbative effect we need multi-instanton corrections. In Fig. 10 we compare the exact ground and first excited state energies with the one- and two-instanton seventh-order approximation—see Figs. 10(a) and 10(b) $-E_{\epsilon, 1,7}$ and $E_{\epsilon, 2,7}$. Multi-instanton corrections account for the nonperturbative gap opening. For the values of $g$ shown, the Meijer- $G$ approximants appear to be well converged in the oneinstanton case, while for the two-instanton case they appear to be well converged up to $g=0.08$ and are likely not inaccurate for all the values of $g$ shown. From our forays at larger instanton orders we suspect that the convergence of the Meijer- $G$ approximants gets slower with increasing instanton order. This is likely due to the fact that the Borel transform removes factorial growth, while the coefficient growth in the divergent series that enters the multi-instanton expansion depends on instanton order, and they can grow superfactorially. Does this trend continue at higher instanton orders? We leave the question of convergence of the summed-up multiinstanton expansion to future work. In any case, this low order calculation shows that multi-instanton contributions open up the gap and that the two-instanton approximation improves on the one-instanton approximation. Once again Meijer- $G$ approximants offer an economical, yet accurate, approach to evaluate these corrections.

With the summed-up one- and two-instanton approximations we can discuss the cancellation of the nonperturbative ambiguity as a function of $g$. We know that in the limit $g \rightarrow 0^{+}$the nonperturbative ambiguity is canceled by the two-instanton contribution, and it is left intact by the one-instanton contribution [28]. However with a 
(a)

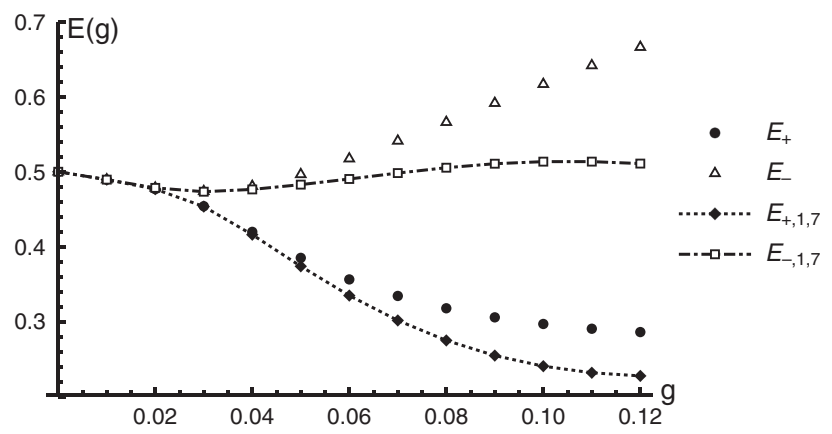

(b)

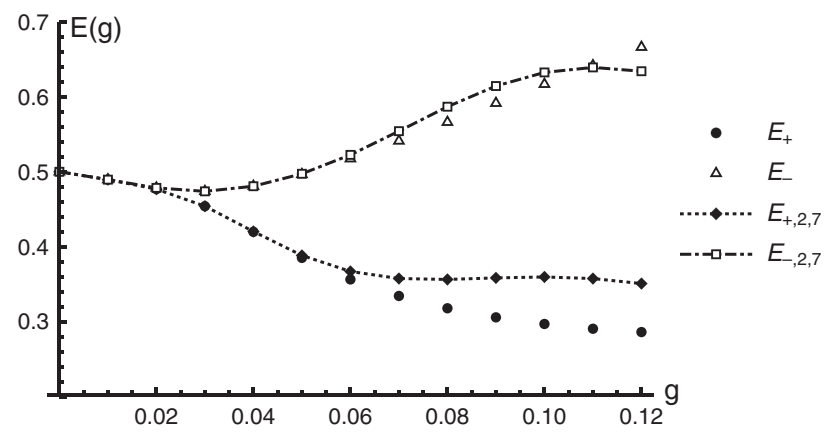

FIG. 10. Summation of the one- and two-instanton contributions to the ground and first excited states of the quantum mechanical double-well potential via seventh-order Meijer- $G$ approximants. (a) Numerically obtained exact result [filled circles (ground state, $E_{+}$) and empty triangles (first excited state, $\left.E_{-}\right)$], together with the one-instanton approximation as calculated by seventh-order Meijer- $G$ approximants for the ground state [dotted line with filled diamonds $\left(E_{+, 1,7}\right)$ ] and first excited state [dot-dashed line with empty squares $\left.\left(E_{-, 1,7}\right)\right]$. (b) The seventh-order Meijer- $G$ two-instanton approximation compared with the exact result. While the Borel sum converges rapidly with increasing approximant order, the one- and two-instanton contributions converge at a slower rate. For $g=0.12$ oneinstanton approximants of order $N \geq 5$ appear well converged. This is not the case for two-instanton Meijer- $G$ approximations that are not fully converged for $N=6-7$.

summed-up multi-instanton expansion the ambiguity cancellation is far from trivial-way more complex than in the previous examples we have discussed. At the two-instanton order there are four different Borel sums, each of which introduces its own ambiguity; exact ambiguity cancellation at the two-instanton order only happens in a rather strict $g \rightarrow 0^{+}$limit, and it has been discussed in detail by ZinnJustin and Jentschura [28]. Here we address the ambiguity cancellation at the two-instanton level, beyond the leading order cancellation present as $g \rightarrow 0^{+}$. In Fig. 11 we show $\operatorname{Im}\left[E_{+}(g)\right]$ as a function of $g$. Away from $g=0$ the ambiguity is reduced but not exactly canceled. It is interesting to note that the one-instanton cancels part of the ambiguity away from $g \rightarrow 0$; away from $g \rightarrow 0^{+}$the two-instanton contribution does not result in a marked improvement relative to the one-instanton result.

In line with the results of previous sections, the Meijer- $G$ approximants are then able to provide good approximations

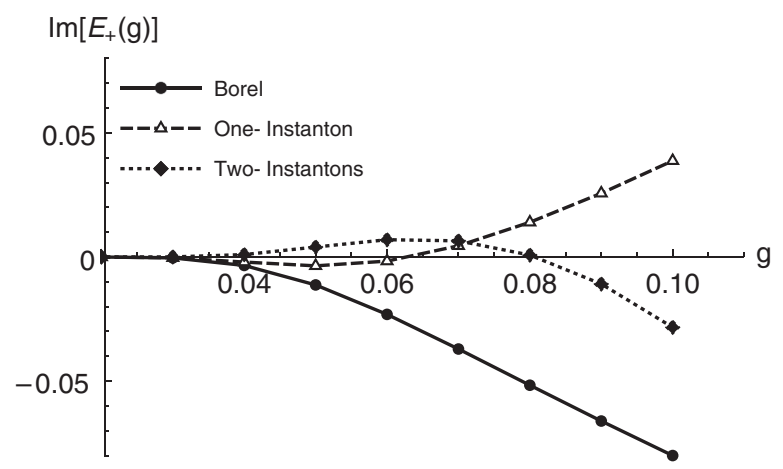

FIG. 11. Nonperturbative ambiguity as a function of coupling strength calculated for the quantum mechanical double-well problem. One- and two-instanton contributions reduce the size of the ambiguity but do not entirely cancel it in the interval $0.03<g<0.1$. to the Borel sum and one-instanton correction, apparently converging quickly with increasing order. We have observed that the convergence of the approximants becomes slower with increasing instanton order. The cancellation of the nonperturbative ambiguity has been studied for $g>0$ and appears fragile: each of the Borel sums performed by Meijer- $G$ summation at each instanton order has its own ambiguity, and the cancellation is not as neat as in the previous examples, where the transseries contained only two terms. Away from $g=0$ the oneinstanton contribution reduces the size of the ambiguity, but the two-instanton contribution does not yield a marked improvement relative to one-instanton calculations. In contrast, as $g \rightarrow 0^{+}$the two-instanton contribution cancels exactly the nonperturbative ambiguity, while the oneinstanton contribution misses this cancellation entirely.

\section{DISCUSSION: IS BOREL-PADÉ OBSOLETE?}

We have put forward a resummation approach that apparently surpasses the commonly used Borel-Padé method in accuracy and ease of use. So a natural question to ask is whether we have actually put the final nail in the coffin of Borel-Padé approaches. The short answer to this question is... not at all. While there are very clear advantages in adopting Meijer- $G$ approximants, there are also some disadvantages that still need to be alleviated. A few of these advantages are the following:

(1) Meijer-G approximants are easily parametrized. The hypergeometric ansatz allows for a swift parametrization of Meijer- $G$ approximants. By approximating the ratios between consecutive Borel-transformed coefficients by a rational function, one parametrizes a hypergeometric function in the Borel plane. The Laplace transform of any hypergeometric function is 
known analytically in terms of Meijer- $G$ functions. The algorithm given in this section is then an easy recipe to transform the coefficients of a divergent perturbation expansion into tables of Meijer- $G$ approximants. One only needs to find the rational functions $r_{N}(n)$ that approximate ratios between consecutive coefficients in the Borel plane and from them the hypergeometric vectors; such a program involves only the solution of a set of linear equations in the former case and the calculation of polynomial roots in the latter case.

(2) Meijer-G approximants are more accurate than Borel-Padé approximants. The examples given here, plus a number of other problems we have considered over the course of this work, lead us to believe that this is clearly the case when the convergence-limiting singularity in the Borel plane is a branch cut. Here we have shown how the Meijer- $G$ approximants actually converge to the exact Borel sum in various examples from QFT.

(3) Meijer-G functions also approximate generalized Borel sums. Why should we be limited to Borel transforms of the form $b_{n}=z_{n} / n$ !? Or Mittag-Leffler transforms of the form $b_{n}=z_{n} / \Gamma(a+b n)$ ? Work in progress shows that more general transforms can be defined that are able to remove, in essence, arbitrary asymptotic coefficient growth and that the resulting approximants can also be represented in terms of Meijer- $G$ functions. Generalizations of the Borel sum can easily be constructed by means of Meijer- $G$ functions.

(4) Hypergeometric summation can be used together with conformal mapping techniques. Conformal mapping in the Borel plane can be combined with Padé summation. It is equally possible to replace Padé summation by hypergeometric summation also in this context, but we have not yet carried out a full study on this subject.

But there are disadvantages too:

(1) Meijer-G approximants are new, and therefore their convergence properties are unknown. In contrast, the properties of Padé approximants are well understood.

(2) Meijer-G approximants can be difficult to evaluate numerically, particularly at large orders. Our approach involves the numerical evaluation of Meijer- $G$ functions, using black boxes which may have not been fully tested at very large orders (who ever needed ${ }_{11} F_{10}$ ?). To obtain accurate Meijer- $G$ approximations one typically needs high-accuracy input data, which may not be available.

(3) Rational approximations in the hypergeometric ansatz can have poles for positive $n$. Typically some of the Meijer- $G$ approximants are affected by these spurious poles. The approximants affected by such poles are generally not accurate.
(4) Padé approximants are better for poles. Meijer- $G$ approximants are very well suited for problems where the Borel plane has a branch cut. But this is not always the case. In some cases the perturbation expansion, or its Borel-transformed counterpart, may have its convergence limited by a pole. Physical examples of such behavior include perturbative spectral functions in Green's function theory [68] and the beta function of supersymmetric Yang-Mills theories [69]. In such cases Padé or Borel-Padé approximants are likely a better summation method than hypergeometric or Borel-hypergeometric summation. Our approach therefore complements, but does not replace, Padé and Borel-Padé summation.

(5) Padé approximants can deal with any singularity structure in the Borel plane provided that their order is sufficiently high, while Meijer- $G$ approximants are designed to work on systems where a single branch cut limits the convergence of the Borel-transformed series. They can also deal with multiple cut systems by a suitable change of variable as, for instance, expansions in powers of $g^{2}$, where the system has a double cut in $g$ but a single cut when the variable is $g^{2}$. In contrast, for sufficiently high orders the zeros and poles of Padé approximants can mimic any branch cut structure, including a multiply cut Borel plane.

Our work then calls for further investigations on the convergence properties of Meijer- $G$ approximants, and extensive stress testing of numerical black boxes for Meijer- $G$ function evaluation. A more fundamental problem is the development of generalized Borel summation methods and parametrizing the corresponding Meijer- $G$ approximants. Finally it will be interesting to see how such a summation approach, based on high-end special functions, would fare in a real-world scenario where only very few coefficients of limited accuracy are available.

\section{CONCLUSIONS}

To conclude, we put forward a simple algorithm that enables a fast and accurate low order Borel summation. The algorithm is a generalized Borel-hypergeometric approach, where the hypergeometric ansatz is used to transform the coefficients of a divergent series into a table of hypergeometric approximants in the Borel plane, and hence into a table of Meijer- $G$ approximants to the Borel sum of the series. We successfully applied this technique to the summation of divergent series and resurgent transseries by Meijer- $G$ approximants. We have considered as examples various partition functions in zero-dimensional QFT; in these cases the Meijer- $G$ approximants converge to the exact answer at order $N=5$. We have used these approximants to sum transseries (Borel-hypergeometric-Écalle sum), completely removing the nonperturbative ambiguity in the Borel sums. The summation of the quartic anharmonic 
oscillator, the calculation of the critical exponents for self-avoiding walks in three dimensions, and the summation of the multi-instanton expansion for the quantum mechanical double-well problem provided more challenging examples, where the Borel-transformed series is not purely hypergeometric. Nevertheless the Meijer- $G$ approximants put forward here were also able to yield accurate low order approximations to the Borel sums involved in these examples.

\section{ACKNOWLEDGMENTS}

H. M. and T. G.P. acknowledge financial support from the CNG center under the Danish National Research Foundation, Project No. DNRF103, and T.G.P. was supported by the QUSCOPE center sponsored by the Villum Foundation. H. M. and B. K. N. were supported by NSF Grant No. CHE 1566074.
[1] E. Caliceti, M. Meyer-Hermann, P. Ribeca, A. Surzhykov, and U. D. Jentschura, Phys. Rep. 446, 1 (2007).

[2] M. Mariño, Fortschr. Phys. 62, 455 (2014).

[3] B. J. Bichsel, M. A. Morrison, N. Shafer-Ray, and E. R. I. Abraham, Phys. Rev. A 75, 023410 (2007).

[4] T. Aoyama, M. Hayakawa, T. Kinoshita, and M. Nio, Phys. Rev. D 91, 033006 (2015).

[5] H. Mera, T. G. Pedersen, and B. K. Nikolić, Phys. Rev. Lett. 115, 143001 (2015).

[6] T. G. Pedersen, H. Mera, and B. K. Nikolić, Phys. Rev. A 93, 013409 (2016).

[7] T. G. Pedersen, S. Latini, K. S. Thygesen, H. Mera, and B. K. Nikolić, New J. Phys. 18, 073043 (2016).

[8] U. D. Jentschura, A. Surzhykov, and J. Zinn-Justin, Phys. Rev. Lett. 102, 011601 (2009).

[9] C. M. Bender and T. T. Wu, Phys. Rev. 184, 1231 (1969); Phys. Rev. Lett. 27, 461 (1971); Phys. Rev. D 7, 1620 (1973).

[10] M. V. Kompaniets and E. Panzer, Phys. Rev. D 96, 036016 (2017).

[11] I. Caprini and J. Fischer, Phys. Rev. D 68, 114010 (2003).

[12] D. J. Gross and V. Periwal, Phys. Rev. Lett. 60, 2105 (1988).

[13] A. Grassi, M. Mariño, and S. Zakany, J. High Energy Phys. 5 (2015) 38.

[14] N. V. Prokof'ev and B. Svistunov, Phys. Rev. B 77, 020408 (2008); R. E. V. Profumo, C. Groth, L. Messio, O. Parcollet, and X. Waintal, Phys. Rev. B 91, 245154 (2015).

[15] G. Alvarez and H. J. Silverstone, J. Phys. Commun. 1, 025005 (2017).

[16] O. Costin and G. V. Dunne, J. Phys. A 51, 04LT01 (2018).

[17] R. B. Dingle, Asymptotic Expansions: Their Derivation and Interpretation (Academic Press, London, 1973).

[18] M. V. Berry and C. J. Howls, Proc. R. Soc. A 434, 657 (1991).

[19] I. Aniceto and R. Schiappa, Commun. Math. Phys. 335, 183 (2015).

[20] I. Aniceto, J. G. Russo, and R. Schiappa, J. High Energy Phys. 03 (2015) 172.

[21] A. Cherman, D. Dorigoni, and M. Ünsal, J. High Energy Phys. 10 (2015) 056.

[22] M. Mariño, Instantons and Large N: An Introduction to Non-Perturbative Methods in Quantum Field Theory (Cambridge University Press, Cambridge, 2015).
[23] R. Couso-Santamaría, M. Mariño, and R. Schiappa, J. Phys. A 50, 145402 (2017).

[24] D. Dorigoni, arXiv:1411.3585.

[25] I. Aniceto, G. Basar, and R. Schiappa, arXiv:1802.10441.

[26] M. P. Heller and M. Spalinski, Phys. Rev. Lett. 115, 072501 (2015).

[27] G. V. Dunne and M. Ünsal, Proc. Sci., LATTICE2015 (2016) 010 [arXiv:1511.05977].

[28] J. Zinn-Justin and U. D. Jentschura, Ann. Phys. (N.Y.) 313, 197 (2004); 313, 269 (2004).

[29] A. Cherman, P. Koroteev, and M. Ünsal, J. Math. Phys. (N.Y.) 56, 053505 (2015).

[30] D. I. Kazakov, O. V. Tarasov, and D. V. Sirkov, Theor. Math. Phys. 38, 9 (1979).

[31] A. A. Vladimirov, D. I. Kazakov, and O. V. Tarasov, Sov. Phys. JETP 50, 521 (1979).

[32] J. C. Le Guillou and J. Zinn-Justin, Phys. Rev. B 21, 3976 (1980).

[33] J. C. Le Guillou and J. Zinn-Justin, J. Phys. Lett. 46, 137 (1985).

[34] R. Guida and J. Zinn-Justin, J. Phys. A 31, 8103 (1998).

[35] M. Y. Nalimov, V. A. Sergeev, and L. Sladkoff, Theor. Math. Phys. 159, 499 (2009).

[36] D. V. Batkovich, M. V. Kompaniets, and K. G. Chetyrkin, Nucl. Phys. B906, 147 (2016).

[37] M. V. Kompaniets, J. Phys. Conf. Ser. 762, 012075 (2016).

[38] H. Kleinert, Path Integrals in Quantum Mechanics, Statistics, and Polymer Physics (World Scientific, Singapore, 1995).

[39] P. M. Stevenson, Phys. Rev. D 23, 2916 (1981).

[40] P. A. Baikov, K. G. Chetyrkin, and J. H. Kühn, J. High Energy Phys. 04 (2017) 119; Phys. Rev. Lett. 118, 082002 (2017).

[41] H. Kleinert and V. Schulte-Frohlinde, Critical Properties of $\phi^{4}$-Theories (World Scientific, Singapore, 2001).

[42] S. Graffi, V. Grecchi, and B. Simon, Phys. Lett. B 32, 631 (1970).

[43] O. Costin, Asymptotics and Borel Summability (CRC Press, Boca Raton, FL, 2008).

[44] S. Graffi and V. Grecchi, J. Math. Phys. (N.Y.) 19, 1002 (1978).

[45] G. A. Baker and P. Graves-Morris, Pad Approximants (Cambridge University Press, Cambridge, 1996). 
[46] H. Mera, T. G. Pedersen, and B. K. Nikolić, Phys. Rev. B 94, 165429 (2016).

[47] S. Sanders and M. Holthaus, New J. Phys. 19, 103036 (2017); J. Phys. A 50, 465302 (2017).

[48] H. Mera, M. Lannoo, C. Li, N. Cavassilas, and M. Bescond, Phys. Rev. B 86, 161404 (2012).

[49] N. Cavassilas, M. Bescond, H. Mera, and M. Lannoo, Appl. Phys. Lett. 102, 013508 (2013).

[50] H. Mera, M. Lannoo, N. Cavassilas, and M. Bescond, Phys. Rev. B 88, 075147 (2013).

[51] M. Bescond, C. Li, H. Mera, N. Cavassilas, and M. Lannoo, J. Appl. Phys. 114, 153712 (2013).

[52] NIST Digital Library of Mathematical Functions, https:// dlmf.nist.gov.

[53] A. Erdèlyi, W. Magnus, F. Oberhettinger, and F. G. Tricomi, Higher Transcendental Functions Vol. I (McGraw-Hill, New York, 1953).

[54] H. Kleinert, S. Thoms, and W. Janke, Phys. Rev. A 55, 915 (1997).

[55] F. Jasch and H. Kleinert, J. Math. Phys. (N.Y.) 42, 52 (2001).

[56] W. Janke and H. Kleinert, Phys. Rev. Lett. 75, 2787 (1995).

[57] V. I. Yukalov, J. Math. Phys. (N.Y.) 32, 1235 (1991).
[58] V. I. Yukalov and E. P. Yukalova, Ann. Phys. (N.Y.) 277, 219 (1999).

[59] F. H. Stillinger, J. Chem. Phys. 70, 4067 (1979).

[60] F. H. Stillinger and T. A. Weber, Phys. Rev. A 27, 2642 (1983).

[61] R. Beals and J. Szmigielski, Not. Am. Math. Soc. 60, 866 (2013).

[62] O. I. Marichev, Handbook of Integral Transform of Higher Transcendental Functions (Wiley, New York, 1983); V. Adamchik and O. I. Marichev, in Proceedings of the 1990 International Symposium on Symbolic and Algebraic Computation, edited by S. Watanabe and M. Nagata (ACM, Academic Press, New York, 1990).

[63] G. H. Hardy, Divergent Series (Oxford University Press, New York, 1949).

[64] L. Pollet, N. V. Prokof'ev, and B. V. Svistunov, Phys. Rev. Lett. 105, 210601 (2010).

[65] F. J. Dyson, Phys. Rev. 85, 631 (1952).

[66] N. Clisby, J. Phys. A 50, 264003 (2017); N. Clisby and B. Dünweg, Phys. Rev. E 94, 052102 (2016).

[67] M. Marucho, J. Math. Phys. (N.Y.) 49, 043509 (2008).

[68] Y. Pavlyukh, Sci. Rep. 7, 504 (2017).

[69] V. A. Novikov, M. A. Shifman, A. I. Vainshtein, and V. I. Zakharov, Nucl. Phys. B229, 381 (1983). 